TRANSACTIONS OF THE

AMERICAN MATHEMATICAL SOCIETY

Volume 364, Number 6, June 2012, Pages 2959-2985

S 0002-9947(2012)05386-4

Article electronically published on January 26, 2012

\title{
THE STRUCTURE AND DEFINABILITY IN THE LATTICE OF EQUATIONAL THEORIES OF STRONGLY PERMUTATIVE SEMIGROUPS
}

\author{
MARIUSZ GRECH
}

\begin{abstract}
In this paper, we study the structure and the first-order definability in the lattice $\mathcal{L}(S P)$ of equational theories of strongly permutative semigroups, that is, semigroups satisfying a permutation identity

$$
x_{1} \cdots x_{n}=x_{\sigma(1)} \cdots x_{\sigma(n)}
$$

with $\sigma(1)>1$ and $\sigma(n)<n$. We show that each equational theory of such semigroups is described by five objects: an order filter, an equivalence relation, and three integers. We fully describe the lattice $\mathcal{L}(S P)$; inclusion, operations $\vee$ and $\wedge$, and covering relation. Using this description, we prove, in particular, that each individual theory of strongly permutative semigroups is definable, up to duality.
\end{abstract}

\section{INTRODUCTION}

In 22, 16, A. Tarski and R. McKenzie raised problems of first-order definability in the lattices of equational theories. In a series of papers 8, 9, 10, 11, J. Ježek has solved the most general problems in this area considering the case of lattices of equational theories of a given type. In [12 Ježek and McKenzie have studied the first-order definability in the lattice of equational theories of semigroups. They have proved that in this lattice many interesting sets of theories are definable (e.g. the finitely axiomatizable theories, the locally finite theories, and the theories of finite semigroups). Moreover, they have proved that each finitely axiomatizable locally finite theory is individually definable up to duality (i.e., up to inverting the order of occurrence of letters in defining equations). They conjecture that the assumption on local finiteness in the last quoted result may be omitted and that, in consequence, the lattice in question has no nontrivial automorphisms, but these problems still remain open.

One of the reasons that the problems above seem very hard is that we have very little detailed knowledge about the structure of the lattice of equational theories of semigroups. In contrast, we have very good knowledge about the structure of the lattice $L(\mathrm{Com})$ of equational theories of commutative semigroups ([13] and [7, 4]). Using this, in 14, A. Kisielewicz has proved that many sets and individual theories in $L(C o m)$ are definable. However, he also discovered that there exist nontrivial automorphisms of $L(\mathrm{Com})$.

Received by the editors May 26, 2010.

2000 Mathematics Subject Classification. Primary 03C07; Secondary 03C05, 08B15.

Key words and phrases. Strongly permutative semigroup, lattice, equational theories.

The author was suported in part by Polish KBN grant 4319/PB/JM/10. 
In [6], we have completed the study of the first-order definability in the lattice $L(C o m)$. We have obtained complete descriptions of the set of definable theories and of the group of automorphisms of $L(\mathrm{Com})$, which has appeared to form an uncountable Boolean group.

It seems natural to now consider the automorphism groups of intermediate classes of equational theories of semigroups to find some "minimal examples" of lattices with small automorphism groups.

A permutative semigroup is one satisfying a permutation identity of the form

$$
x_{1} \cdots x_{n}=x_{\sigma(1)} \cdots x_{\sigma(n)}
$$

for some nontrivial permutation $\sigma$. Perkins [18] proved that such a semigroup necessarily satisfies an equation of the form

$$
x_{1} \cdots x_{k} y z x_{k+1} \cdots x_{n}=x_{1} \cdots x_{k} z y x_{k+1} \cdots x_{n},
$$

and therefore he used the name almost commutative for such semigroups.

The set of equational theories of permutative semigroups forms a lattice that contains the lattice of equational theories of commutative semigroups. An extension of Kisielewicz's 13 description to the lattice of all equational theories of permutative semigroups does not seem easy. The main obstacle is that, as it was proved in [18, there are equational theories of permutative semigroups that fail to be finitely based. In contrast, it is well known (see [18, 20, for instance) that every theory containing an equation (11) for some permutation $\sigma$ with $\sigma(1)>1$ and $\sigma(n)<n$ is finitely based. Such theories (and corresponding semigroups) will be called strongly permutative. It is known (see e.g. 20]) that for every strongly permutative theory $T$ there exists $m>1$ such that $T$ contains all permutation equations (11) for all $n \geq m$.

If we wish to study first-order definability of intermediate lattices of equational theories of semigroups, the case of all strongly permutative semigroups may be considered as an appropriate candidate for the beginning. Every strongly permutative variety is finitely based, and therefore the set of all strongly permutative varieties is countable.

For $k \geq 0$ and $n>1$, let $B_{k, n}$ denote the set that consists of $n$ ! identities of the following form:

$$
t_{1} \cdots t_{k} x_{1} x_{2} \cdots x_{n} t_{k+1} \cdots t_{2 k}=t_{1} \cdots t_{k} x_{\sigma(1)} x_{\sigma(2)} \cdots x_{\sigma(n)} t_{k+1} \cdots t_{2 k},
$$

where $\sigma$ is a permutation on the set $\{1, \ldots, n\}$. If $k \geq 0$, then an equational theory $T$ is called $k$-permutative if for some $n \geq 2, T=B_{k, n}([21)$. By the result of Perkins [18, each permutative theory is $k$-permutative for some $k \geq 0$. For each $n$, the set of all $k$-permutative theories with $k \leq n$ forms a sublattice of the lattice $P$ of all permutative semigroup theories, which we denote by $P(n)$. It is easy to see that for each $n \geq 0$, we have $P(n) \subset P(n+1)$. It is also easy to see that the term "strongly permutative variety" is equivalent to the term "0-permutative variety". Our result can be considered as the first step in proving the following conjecture, which we believe may be true.

Conjecture. For each $n \geq 0$ and $k \leq n$, every finitely based $k$-permutative theory is individually first-order definable, up to duality, in the lattice $P(n)$.

The first part of this paper is aimed to prepare a general machinery to work with strongly permutative theories, which we believe gives a direct insight and may 
be useful to formulate and prove further results in the area. This generalizes, in fact, the tools worked out in [13] and results in [4, 7] for commutative semigroups. It seems that it may be the largest part of the lattice of equational theories of all semigroups admitting such a nice description.

The second part, starting from section 7, is devoted to proving the result that every equational theory of strongly permutative semigroups is individually firstorder definable, up to duality (Theorem 10.9).

\section{Preliminaries}

It was J. Almeida [1] who discovered that behind the lattice of varieties of commutative semigroups a combinatorial structure of better-quasi-orderings is hidden. This also can be extended into the strongly permutative case. Once it is not necessery to prove the results in the second part of the paper, it may be useful for attacking more difficult problems in the area.

2.1. Better-quasi-orderings. Here we recall a definition of a better-quasi-ordering (well-quasi-ordering) and some results from [6, 17.

Let $\omega$ be the set of nonnegative integers with the usual order. For $s, t \subseteq \omega$ we write $s \leq t(s<t)$ if $s$ is a (proper) initial segment of $t$.

Define $s \triangleleft t$ to hold if there are $n>0$ and $i_{0}<\cdots<i_{n} \in \omega$ such that for some $m<n, s=\left\{i_{0}, \ldots, i_{m}\right\}$ and $t=\left\{i_{1}, \ldots, i_{n}\right\}$.

For an infinite set $X \subseteq \omega$, a set $B$ of finite subsets of $X$ will be called a barrier on $X$ if the following conditions hold:

(i) $\emptyset \notin B$,

(ii) for every infinite $Y \subseteq X$ there is an $s \in B$ such that $s<Y$,

(iii) if $s, t \in B$ and $s \neq t$, then $s \nsubseteq t$.

Let $(Q, \leq)$ be a quasi-ordering. For a barrier $B$, a function $f: B \rightarrow(Q, \leq)$ is $\operatorname{good}$ if there are $s, t \in B$ such that $s \triangleleft t$ and $f(s) \leq f(t)$, and $f$ is bad otherwise. Then, $(Q, \leq)$ is a better-quasi-ordering (bqo) if for every barrier $B$ and every $f: B \rightarrow$ $(Q, \leq), f$ is good. The quasi-ordering $(Q, \leq)$ is a well-quasi-ordering (wqo) if for every barrier $B$ consisting of one-element sets and every $f: B \rightarrow(Q, \leq), f$ is good.

It is an immediate consequence of definition that if $(Q, \leq)$ is bqo, then $(Q, \leq)$ is wqo. There is also another characterization of well-quasi-orderings. A quasiordering $(Q, \leq)$ is a well-quasi-ordering if and only if every order filter is finitely generated.

For the quasi-orderings $\left(Q_{i}, \leq_{i}\right), i \in\{1, \ldots, n\}$, we define $\bigcup\left(Q_{i}, \leq_{i}\right)$ to be a quasi-ordering $\left(\bigcup Q_{i}, \leq\right)$, where $p \leq q$ if and only if there is $i$ such that $p, q \in Q_{i}$, and $p \leq_{i} q$.

Proposition 2.1 ([17]). If $\left(Q_{i}, \leq_{i}\right), i \in\{1, \ldots, n\}$, are bqo, then $\bigcup\left(Q_{i}, \leq_{i}\right)$ is bqo.

Below, we write three easy facts which can be found in [6].

Fact 2.2. Let $(Q, \leq)$ be bqo and $Q_{1} \subseteq Q$. Then $\left(Q_{1}, \leq\right)$ is bqo.

Fact 2.3. Let $\left(Q, \leq_{1}\right),\left(Q, \leq_{2}\right)$ be quasi-orderings such that for $q, w \in Q$, if $q \leq_{1} w$, then $q \leq_{2} w$. Then, if $\left(Q, \leq_{1}\right)$ is bqo, then $\left(Q, \leq_{2}\right)$ is bqo.

Fact 2.4. For quasi-orderings $\left(Q_{1}, \leq_{1}\right),\left(Q_{2}, \leq_{2}\right)$ let $f: Q_{1} \rightarrow Q_{2}$ be an onto mapping such that for $q, w \in Q_{1}$, if $q \leq_{1} w$, then $f(q) \leq_{2} f(w)$. Then, if $\left(Q_{1}, \leq_{1}\right)$ is bqo, then $\left(Q_{2}, \leq_{2}\right)$ is bqo. 
Moreover, the following fact can be easily verified.

Fact 2.5. For quasi-orderings $\left(Q_{1}, \leq_{1}\right),\left(Q_{2}, \leq_{2}\right)$ let $f: Q_{1} \rightarrow Q_{2}$ be a mapping such that for $q, w \in Q_{1}$, we have $q \leq_{1} w$ if and only if $f(q) \leq_{2} f(w)$. Then, if $\left(Q_{2}, \leq_{2}\right)$ is bqo, then $\left(Q_{1}, \leq_{1}\right)$ is bqo.

2.2. The lattices $\mathcal{L}(\mathrm{Com})$. Now we recall the terminology and facts concerning the description of the lattice of equational theories of commutative semigroups $\mathcal{L}(\mathrm{Com})$ based on [13, taking into account some improvements from [4, 7, 5, We recall only those facts that we apply in this paper. For more details the reader is referred to [13, 7, 4, 5] and to the introductions in [14, 6].

Generally, an equational theory of commutative semigroups is determined by four parameters $J, m, r, \pi$, where $m, r$ are integers, $J$ is an order filter in a certain ordered set of finite sequences of integers, and $\pi$ is a special additional set of equations of finite character (called the remainder).

Let $\Gamma$ be the set of all finite sequences $\left(a_{1}, \ldots, a_{n}\right)$ of nonnegative integers such that at least one $a_{i} \neq 0$, and let $\Gamma_{+}$consist of those sequences where $a_{i}>0$ for all $i$. For a sequence $\mathbf{a}=\left(a_{1}, \ldots, a_{n}\right) \in \Gamma$ and a permutation of the indices $p \in S_{n}$, we put $p(\mathbf{a})=\left(a_{p(1)}, \ldots, a_{p(n)}\right)$; if $p \in S_{m}$ with $n \neq m$, then we put $p(\mathbf{a})=\mathbf{a}$. Further, for every $\gamma \geq 0$ we denote $h_{\gamma}(\mathbf{a})=\left(a_{1}, \ldots, a_{n}, \gamma\right)$, and for $1 \leq i, j \leq n$, $i \neq j$, we denote $g_{i j}(\mathbf{a})=\left(a_{1}, \ldots, a_{i}+a_{j}, \ldots, a_{n}\right)$, where the latter is the sequence obtained from a by replacing $a_{i}$ by $a_{i}+a_{j}$ and deleting $a_{j}$; otherwise, we denote $g_{i j}(\mathbf{a})=\mathbf{a}$. By $P G H$ we denote the smallest class of unary operations on $\Gamma$ closed under composition and containing all the operations $h_{\gamma}, g_{i j}$ and permutations $p$.

For sequences $\mathbf{a}, \mathbf{b} \in \Gamma$, we define $\mathbf{a} \leq_{K} \mathbf{b}$ if and only if there exists an operation $f \in P G H$ such that $f(\mathbf{a})=\mathbf{b}$. In [5], the following is proved.

Proposition 2.6 ([5]). The quasi-ordering $\left(\Gamma, \leq_{K}\right)$ is bqo.

For other results in this direction see [1] also.

Proposition 2.6 shows, in particular, that every (order) filter $J$ is finitely generated. The filter generated by the sequences $\mathbf{a}_{1}, \ldots, \mathbf{a}_{t}$ is denoted $\left[\mathbf{a}_{1}, \ldots, \mathbf{a}_{t}\right]$.

Now, a commutative semigroup equation of the form

$$
x_{1}^{a_{1}} \cdots x_{n}^{a_{n}}=x_{1}^{b_{1}} \cdots x_{n}^{b_{n}}
$$

will be identified with the pair $(\mathbf{a}, \mathbf{b})$ of sequences $\mathbf{a}=\left(a_{1}, \ldots, a_{n}\right)$ and $\mathbf{b}=$ $\left(b_{1}, \ldots, b_{n}\right)$, where $a_{i}+b_{i}>0$, and is denoted by $(\mathbf{a}, \mathbf{b})$ for short. Such an equation is nontrivial if $a_{i} \neq b_{i}$ for some $i$. It is clear that for $f \in P G H$, the equation $(f(\mathbf{a}), f(\mathbf{b}))$ is a consequence of $(\mathbf{a}, \mathbf{b})$. An equational theory of a commutative semigroup (a theory) is a set of equations closed onto natural operations: multiplication, substitution, permutation of variables and transitivity of equality.

For a nonempty filter $J$ in $\Gamma$, and integers $m \geq 0, r>0$, we define $E(J, m, r)$ to be the theory given by the set of those equations $(\mathbf{a}, \mathbf{b})$, where $\mathbf{a}=\left(a_{1}, \ldots, a_{n}\right)$, $\mathbf{b}=\left(b_{1}, \ldots, b_{n}\right)$ are such sequences in $J$ that satisfy the following conditions:

(J1) for every $i$, if $a_{i} \neq b_{i}$, then $a_{i}, b_{i} \geq m$,

(J2) for every $i, a_{i} \equiv b_{i}(\bmod r)$.

Moreover, we consider the following set of conditions for equations with $a_{i}, b_{i}>0$, which belong to an equivalence relation $\pi$ on the set $\Gamma_{+} \backslash J$ :

$(\pi 1)$ the conditions $(\mathrm{J} 1),(\mathrm{J} 2)$ hold,

$(\pi 2)$ for every permutation $f \in S_{n},(f(\mathbf{a}), f(\mathbf{b})) \in \pi$, 
$(\pi 3)$ for every $i$ such that $a_{i} \neq b_{i}$, and $\gamma=\min \left(a_{i}, b_{i}\right)$, both $h_{\gamma}(\mathbf{a}), h_{\gamma}(\mathbf{b}) \in J$,

$(\pi 4)$ if $f$ is one of the operations $g_{i j}$ or $h_{1}$, then either $(f(\mathbf{a}), f(\mathbf{b})) \in \pi$ or both $f(\mathbf{a}), f(\mathbf{b}) \in J$.

Now let $m \geq 0, r>0$ be integers, $J$ a nonempty filter contained in $[(m)]$ and $\pi$ an equivalence relation on the set $\Gamma_{+} \backslash J$. If every block of $\pi$ contains sequences of one fixed length (regular equations) and if for all pairs $(\mathbf{a}, \mathbf{b}) \in \pi$ the conditions $(\pi 1)-(\pi 4)$ are satisfied, then $\pi$ is called a remainder of the type $(J, m, r)$. For such a remainder, which may be viewed as a set of equations, we define $E(J, m, r, \pi)$ to be the set of equations $E(J, m, r) \cup \pi$. Then:

Theorem 2.7 ([13]). Every set of equation $E(J, m, r, \pi)$ defined above is a theory, and every equational theory of commutative semigroups, except the theory consisting of trivial equations, is of this form.

Theorem 2.8 ([13]). If $E_{1}=E\left(J_{1}, m_{1}, r_{1}, \pi_{1}\right)$ and $E_{2}=E\left(J_{2}, m_{2}, r_{2}, \pi_{2}\right)$ belong to $\mathcal{L}(C o m)$, then the following hold:

(i) $E_{1} \supseteq E_{2}$ if and only if $J_{1} \supseteq J_{2}, m_{1} \leq m_{2}, r_{1}$ divides $r_{2}$, and $\pi_{1} \cup J_{1}^{2} \supseteq \pi_{2}$.

(ii) $E_{1} \vee E_{2}=E\left(J, \min \left(m_{1}, m_{2}\right), \operatorname{gcd}\left(r_{1}, r_{2}\right), \pi\right)$, where $J \supseteq J_{1} \cup J_{2}$, and $\pi$ is a minimal equivalence relation on the set $\Gamma_{+} \backslash J$ that contains both the relations $\pi_{1}$ and $\pi_{2}$ restricted to the set $\Gamma_{+} \backslash J$.

(iii) $E_{1} \wedge E_{2}=E\left(J_{1} \cap J_{2}, \max \left(m_{1}, m_{2}\right), \operatorname{lcm}\left(r_{1}, r_{2}\right), \pi\right)$, where $\pi$ is an equivalence relation on the set $\Gamma_{+} \backslash\left(J_{1} \cap J_{2}\right)$ contained in $\left(\pi_{1} \cap \pi_{2}\right) \cup\left(\pi_{1} \cap J_{2}^{2}\right) \cup\left(\pi_{\cap} J_{1}^{2}\right)$.

The following remarks will be used throughout this paper.

Remark 2.9 ([13]). A theory $E(J, m, r, \pi)$ consists of those equations $(\mathbf{a}, \mathbf{b})$ for which either $\mathbf{a}, \mathbf{b} \in J$ and satisfy the conditions (J1) and (J2) or $\mathbf{a}, \mathbf{b} \notin J$ and $(\mathbf{a}, \mathbf{b}) \in \pi$. Moreover:

(i) if $\mathbf{a} \leq_{K} \mathbf{b}$, then $\mathbf{a}, \mathbf{b} \in J$,

(ii) if $(\mathbf{a}, \mathbf{b})$ is an irregular equation, then $\mathbf{a}, \mathbf{b} \in J$,

(iii) if $(\mathbf{a}, \mathbf{b}) \in \pi$, then $(\mathbf{a}, \mathbf{b})$ is a regular equation and the sequences $\mathbf{a}, \mathbf{b}$ are incomparable or equivalent in the better-quasi-ordering $\left(\Gamma_{+}, \leq_{K}\right)$.

Remark 2.10 ([4 Theorem 2.5]). For every remainder $\pi$ there is a finite set $\bar{\pi}$ of the blocks of $\pi$ such that every block $C \in \pi$ is of the form $f(B)$ for some $B \in \bar{\pi}$ and $f \in P H$.

\section{QUASI-ORDERINGS $\leq_{n}$}

Let $\mathcal{W}=F(X)$ be the free semigroup over countable alphabet $X=\left\{x_{1}, x_{2}, \ldots\right\}$ for $i \in\{1, \ldots, t\}$. The elements of $X$ are called letters or variables. The elements of $\mathcal{W}$ are called words and will be written in the form

$$
v=y_{1} y_{2} \ldots y_{t},
$$

where $y_{i} \in X$ for all $i$. The support of $v$, written $\operatorname{supp}(v)$, is the set of variables occurring in $v$. The length of $v$ is $t$, and we write $|v|=t$.

Moreover, $|v|_{x}$ is the number of occurrences of the letter $x$ in $v$. The notation $v=v\left(x_{1}, \ldots, x_{k}\right)$ means that $\operatorname{supp}(v) \subseteq\left\{x_{1}, \ldots, x_{k}\right\}$. We also write $x^{t}$ to denote the word $x x \cdots x$ of length $t$.

We introduce some operations on $\mathcal{W}$. By

$$
r_{y}(v)=v y \text { and } l_{y}(v)=y v
$$


we denote adjoining an additional variable $y$ to the word $v$, on the right and on the left, respectively. Usually we will assume that $y$ does not occur in $v$. For identifying variables we introduce

$$
g_{x_{i} x_{j}}(v)=v\left(x_{1}, \ldots, x_{j-1}, x_{i}, x_{j+1}, \ldots, x_{k}\right) \text { if } 1 \leq i<j \leq k .
$$

We put $g_{x_{j} x_{i}}(v)=g_{x_{i} x_{j}}(v)$ for $j<i$. For substitution we write

$$
t_{x_{i} y}(v)= \begin{cases}v\left(x_{1}, \ldots, x_{i-1}, x_{i} y, x_{i+1}, \ldots, x_{k}\right), & \text { if } 1 \leq i \leq k, \\ v & \text { otherwise }\end{cases}
$$

Finally, for a permutation $p \in S_{l}$, we define

$$
p(v)= \begin{cases}v\left(x_{p(1)}, \ldots, x_{p(l)}\right), & \text { if } l \geq k \\ v & \text { otherwise. }\end{cases}
$$

We define $P$ to be the set of all operations of the form (5). By $\mathcal{O}$, we denote the set of all operations on $\mathcal{W}$ generated by operations (2) $-(5)$. We define a binary relation $\leq_{n}$ on the set $\mathcal{W}$. Let $v, w \in \mathcal{W}$. Assume that $\operatorname{supp}(v) \cup \operatorname{supp}(w) \subseteq$ $\left\{x_{1}, \ldots, x_{k}\right\}$ for $k>0$. We write $w \leq_{n} v$ if either there is an operation $f \in \mathcal{O}$ such that $f(v)=w$ or for the sequences $\left(|v|_{x_{1}}, \ldots,|v|_{x_{k}}\right),\left(|w|_{x_{1}}, \ldots,|w|_{x_{k}}\right)$ we have $\sum_{i=1}^{k}|w|_{x_{i}} \geq n$ and $\left(|v|_{x_{1}}, \ldots,|v|_{x_{k}}\right) \leq_{K}\left(|w|_{x_{1}}, \ldots,|w|_{x_{k}}\right)$. The latter is equivalent with the fact that there is an operation $f \in \mathcal{O}$ such that $\left(|f(v)|_{x_{1}}, \ldots,|f(v)|_{x_{k}}\right) \equiv_{K}$ $\left(|w|_{x_{1}}, \ldots,|w|_{x_{k}}\right)$.

Lemma 3.1. Let $v, w \in \mathcal{W}$ be such that $\operatorname{supp}(v) \cup \operatorname{supp}(w) \subseteq\left\{x_{1}, \ldots, x_{k}\right\}$, and there is an operation $f \in \mathcal{O}$ such that $f(v)=w$. Then the following inequality holds: $\left(|v|_{x_{1}}, \ldots,|v|_{x_{k}}\right) \leq_{K}\left(|w|_{x_{1}}, \ldots,|w|_{x_{k}}\right)$.

Proof. For the operations $r_{y}, l_{y}, g_{x_{i} x_{j}}, t_{x_{i} y}, p$, we have an induced action of those operations on the set $\Gamma$. In these actions, the operations are equal to $h_{1}, h_{1}, g_{i j}, h_{|v|_{x_{i}}}$, and $p$, respectively. Now, the statement is obvious.

It is an immediate consequence of the definition and Lemma 3.1 that the relation $\leq_{n}$ is transitive. Hence, $\left(\mathcal{W}, \leq_{n}\right)$ is a quasi-ordering. We will write $v<_{n} w$ if $v \leq_{n} w$ and $w \leq_{n} v$. Moreover, we write $v \equiv_{n} w$ if $v \leq_{n} w$ and $w \leq_{n} v$.

Lemma 3.2. Let $v, w \in \mathcal{W}$.

(i) If $v \leq_{n} w$, then $|v| \leq|w|$.

(ii) If $v<_{n} w$, and $\operatorname{supp}(v)=\operatorname{supp}(w)$, then $|v|<|w|$.

(iii) $v \equiv_{n} w$ if and only if there is $k \in \mathbb{N}_{+}$and $p \in S_{k}$ such that $p(v)=w$. In particular, $|v|=|w|$.

Proof. All of (i), (ii) and (iii) are true when $|w| \geq n$ (see 4 , for instance). Thus, we assume that $f(v)=w$ for some $f \in \mathcal{O}$. For (i), it is enough to observe that every operation (2) - (5) does not decrease the length of the words. For (ii), observe that the operations that do not increase the length of the words are $g_{x_{i} x_{j}}$ and permutations. It is clear that when we apply a permutation to a word, we obtain an equivalent word. Applying an operation $g_{x_{i} x_{j}}$, we decrease the cardinality of the support. Hence, $f(v)=w$, where $f \in \mathcal{O}$ is a composition of operations of the form (2) - (5) such that at least one of the operations of the form (2) or (41) is used. It implies that $|v|<|w|$. By (i), if $v \equiv_{n} w$, then $|v|=|w|$. Let $f_{1}, f_{2} \in \mathcal{O}$ be such that $f_{1}(v)=w$ and $f_{2}(w)=v$. Since the operations (2) and (4) increase the length of the words, $f_{1}$ and $f_{2}$ are the compositions of the operations $g_{x_{i} x_{j}}$ and permutations. Since the operations $g_{x_{i} x_{j}}$ decrease the cardinality of supports, $f_{1}$ 
must be a composition of permutations. It is clear that this composition is equal to $p$, for some $p \in P$. This proves (iii).

Let $\Gamma_{n}$ be the subset of $\Gamma$ consisting of the sequences with the sum of elements greater than or equal to $n$. By Proposition 2.6 and Fact 2.2, $\left(\Gamma_{n}, \leq_{K}\right)$ is bqo. Let $\mathcal{W}_{n}$ be the subset of $\mathcal{W}$ consisting of the words of the length at least $n$. There is a natural mapping $\phi:\left(\mathcal{W}_{n}, \leq_{n}\right) \rightarrow\left(\Gamma_{n}, \leq_{K}\right), \phi(w)=\left(|w|_{x_{1}}, \ldots,|w|_{x_{t}}\right)$, where $t$ is a minimal number such that $\operatorname{supp}(w) \subseteq\left\{x_{1}, \ldots, x_{t}\right\}$. Obviously, for $w, v \in \mathcal{W}$, we have $w \leq_{n} v$ if and only if $\phi(w) \leq_{K} \phi(v)$. Hence, by Fact 2.5. $\left(\mathcal{W}_{n}, \leq_{n}\right)$ is bqo. The set of the words that belong to $\mathcal{W}$ and have length smaller than $n$, with the quasi-ordering $\leq_{n}$, consists of finitely many equivalence blocks at the relation $\equiv_{n}$. Each equivalence block is trivially bqo. From the facts above and by Proposition 2.1 and Fact 2.3, we have

Theorem 3.3. The quasi-ordering $\left(\mathcal{W}, \leq_{n}\right)$ is bqo. Consequently, every order filter in $\left(\mathcal{W}, \leq_{n}\right)$ is finitely generated.

\section{Sublattice $\mathcal{L}_{S}(S P)$}

By an equational theory of semigroup $T$, we mean the set of equations of the form

$$
v=w
$$

$v, w \in W$, closed under natural operations: substitutions, identifying variables, multiplication by variables and transitivity of equality (i.e., preserved by all the operations from $\mathcal{O}$ and by transitivity of equality). If there is $n$ such that, for every permutation $\sigma \in S_{n}$, an equation

$$
x_{1} x_{2} \cdots x_{n}=x_{\sigma(1)} x_{\sigma(2)} \cdots x_{\sigma(n)}
$$

belongs to $T$, then $T$ is a theory of strongly permutative semigroups. We recall that this is equivalent to the fact that $T$ contains at least one equation of the form (77), for some $n$, such that $\sigma(1)>1$ and $\sigma(n)<n$.

The set of all equational theories of strongly permutative semigroups forms a lattice $\mathcal{L}(S P)$. The least element in $\mathcal{L}(S P)$ is the theory $\mathcal{I}$ consisting of the trivial equations. The greatest theory is the theory generated by the equation $x=y$ (the set of all equations).

Let $e$ be an equation of the form $v=w$. Alternatively, we denote this equation as $(v, w)$ and write

$$
e=(v, w) .
$$

We define $m(e)$ to be the least of the integers $|v|_{x},|w|_{x}$, where $x$ runs over the set of those $x \in \operatorname{supp}(v) \cup \operatorname{supp}(w)$ for which $|v|_{x} \neq|w|_{x}$. If this set is empty, we put $m(e)=\infty$. For a theory $T$, by $m(T)$ we denote the least of the integers $m(e)$, with $e \in T$. Similarly, by $r(e)$ we denote the least of the integers $\left.|| v\right|_{x}-|w|_{x} \mid$, where $x$ runs over the same set as above. Again, if this set is empty, then we denote $r(e)=\infty$. For a theory $T$, by $r(T)$ we denote the least of the integers $r(e)$, with $e \in T$.

Now, let $n=n(T)$ be the last number such that for all $\sigma \in S_{n}$, the equation of the form (17) belongs to a theory $T \in \mathcal{L}(S P)$. Then, by $T_{n}$, we denote the union of the set of all equations of the form (6) such that $|v| \geq n$ and $|w| \geq n$ with the set of all the equations that belong to the theory $\mathcal{I}$. It is easy to see that the set $T_{n}$ is a 
theory that belongs to $\mathcal{L}(S P)$. Moreover, it is obvious that the theory $T_{n}$ belongs to $\mathcal{L}(\mathrm{Com})$. Hence, there are parameters: an order filter $J_{n} \subseteq \Gamma, m \in \mathbb{N}_{+}, r \in \mathbb{N}$, and an equivalence relation $\pi_{n}$ on the set $\Gamma_{+} \backslash J_{n}$ such that $T_{n}=E\left(J_{n}, m, r, \pi_{n}\right)$. The set $J_{n}$ we may identify with the order filter of those words $v\left(x_{1}, \ldots, x_{k}\right) \in \mathcal{W}$ such that $\left(|v|_{x_{1}}, \ldots,|v|_{x_{k}}\right) \in J_{n}$. It may happen that $T_{n}=\mathcal{I}$. In this situation, we have $J_{n}=\emptyset$ and $\pi=\mathcal{I}$. Then we will write $m=\infty$ and $r=\infty$.

Lemma 4.1. Let $T \in \mathcal{L}(S P)$ and $T_{n}=E\left(J_{n}, m, r, \pi_{n}\right)$. Then we have the following:

(i) $m(T)=m$,

(ii) $r(T)=r$.

Moreover, $r$ divides $r(e)$ for every equation $e \in T$.

Proof. We have to show that for the equation $e$ of the form (6), if $e \in T$, then $m(e) \geq m$ and $r$ divides $r(e)$. Let $y \notin \operatorname{supp}(v) \cup \operatorname{supp}(w)$, and consider the equation $e_{n}$ of the form $v_{n}=w_{n}$, where $v_{n}=v y^{n}$ and $w_{n}=w y^{n}$. Obviously, the equation $e_{n}$ belongs to $T_{n}$. Hence, $m\left(e_{n}\right) \geq m$ and $r$ divides $r(e)$. Moreover, $m\left(e_{n}\right)=r(e)$ and $r\left(e_{n}\right)=r(e)$. This proves the lemma.

We define $J(T)$ to be the set consisting of the words $v$ such that an equation $v=w$ belongs to $T$ for some $w \in J_{n}$.

Lemma 4.2. The set $J(T)$ is an order filter on the bqo $\left(\mathcal{W}, \leq_{n}\right)$.

Proof. Let $v \in J$ and $u \geq_{n} v$. Then there is an operation $f \in \mathcal{O}$ such that either $f(v)=u$ or $\left(|f(v)|_{x_{1}}, \ldots,|f(v)|_{x_{k}}\right) \equiv_{K}\left(|u|_{x_{1}}, \ldots,|u|_{x_{k}}\right)$ and $|u| \geq n$. In the latter case the equation $f(v)=u$ belongs to $T_{n}$. Take a word $w \in J_{n}$ such that an equation $v=w$ belongs to $T$. Obviously, equation $f(v)=f(w)$ belongs to $T$. Moreover, $J_{n}$ is an order filter. Hence, $f(w) \in J_{n}$. Consequently, $u \in J$ and $J$ is an order filter.

Let $v \in J$ and $m(v)=\max \left\{|v|_{x}: \quad x \in \operatorname{supp}(v)\right\}$. We define $m(J)$ to be $\min \{m(v): v \in J\}$.

Lemma 4.3. Let $T \in A$. Then $m(J(T)) \geq m(T)$.

Proof. Let $v \in J(T)$ be a word such that $m(v)=m(J(T))$. If $v \in J_{n(T)}(T)$, then $m(v) \geq m(T)$, by assumption on $J_{n(T)}(T)$. If $v \notin J_{n(T)}(T)$, then there is $w \in J_{n(T)}(T)$ such that the equation $v=w$ belongs to $T$. Moreover, there is a variable $x \in \operatorname{supp}(v) \cup \operatorname{supp}(w)$ such that $|v|_{x} \neq|w|_{x}$. Hence, $|v|_{x} \geq m(T)$. Obviously, $|v|_{x} \leq m(v)$. This implies the statement.

Obviously, if $v \in J(T)$ and an equation $w=v$ belongs to $T$, then $w \in J(T)$. By Theorem 3.3, we know that $J(T)$ is generated by a finite set of words.

For the equation $e$ of the form (6), we consider two conditions:

(J1) $m(e) \geq m$,

(J2) $r(e)$ is divisible by $r$.

It is easy to observe that the conditions (J1) and (J2) generalize the corresponding conditions from subsection 2.2. In the sequel, every reference to (J1) and (J2) concerns conditions formulated above.

Lemma 4.4. Let $v, w \in J$. Then an equation e of the form $v=w$ belongs to $T$ if and only if e satisfies the conditions (J1) and (J2) with $m=m(T)$ and $r=r(T)$. 
Proof. The "only if" part is an immediate consequence of Lemma 4.1 For the "if" part, we assume that $e$ satisfies the conditions (J1) and (J2). By assumption, there are words $v_{n}, w_{n} \in J_{n}$ such that equations $v=v_{n}$ and $w=w_{n}$ belong to $T$. Let $x \in \operatorname{supp}\left(v_{n}\right) \cup \operatorname{supp}\left(w_{n}\right)$ be such that $\left|v_{n}\right|_{x} \neq\left|w_{n}\right|_{x}$. Then, either $\left|v_{n}\right|_{x} \neq|v|_{x}$ or $|v|_{x} \neq|w|_{x}$, or else $|w|_{x} \neq\left|w_{n}\right|_{x}$. In all these cases $\min \left\{|v|_{x},|w|_{x},\left|v_{n}\right|_{x},\left|w_{n}\right|_{x}\right\} \geq m$, and obviously, $r$ divides the numbers $\left|v_{n}\right|_{x}-|v|_{x},|v|_{x}-|w|_{x}$, and $|w|_{x}-\left|w_{n}\right|_{x}$. Hence, $r$ divides $\left|v_{n}\right|_{x}-\left|w_{n}\right|_{x}=\left|v_{n}\right|_{x}-|v|_{x}+|v|_{x}-|w|_{x}+|w|_{x}-\left|w_{n}\right|_{x}$. This implies that the equation $v_{n}=w_{n}$ satisfies the conditions (J1) and (J2). By Remark 2.9, the equation $v_{n}=w_{n}$ belongs to $T$. Consequently, the equation $e$ belongs to $T$.

If a word $x_{1} \cdots x_{k}$ belongs to the filter $J$, then by Lemma 4.2 for every $\sigma \in S_{k}$, $x_{\sigma(1)} \cdots x_{\sigma_{k}}$ belongs to $J$, too. Moreover, it is easy to see that, for every $\sigma \in S_{k}$, the equation $x_{1} \cdots x_{k}=x_{\sigma(1)} \cdots x_{\sigma_{(k)}}$ belongs to $T$. In addition, by Lemma 4.4, we have the following.

Theorem 4.5. For every $T \in \mathcal{L}(S P)$, the word $x_{1} \cdots x_{n(T)-1}$ does not belong to $J(T)$.

For the parameters $n, J, m, r$, where $m \in \mathbb{N}, n, r \in \mathbb{N}_{+}$and $J$ is an order filter on the bqo $\left(\mathcal{W}, \leq_{n}\right)$ such that $m(J) \geq m$ and no word $x_{1} \cdots x_{n-1}$ belongs to $J$, we define an equivalence relation $S$ on the set $\mathcal{L}(S P)$ as follows. A theory $T$ belongs to the class $S(n, J, m, r)$ if $n(T)=n, J(T)=J, m(T)=m, r(T)=r$.

By $T(n, J, m, r)$ we denote the least element of the class $S(n, J, m, r)$.

Theorem 4.6. Let $n>0, m \geq 0, r>0$ and $J$ be an order filter on bqo $\left(W, \leq_{n}\right)$ such that $m(J) \geq m$, and the word $x_{1} \cdots x_{n-1}$ does not belong to $J$. Then theory $T(n, J, m, r)$ consists of those equations e of the form $v=w$ for which either $v$ and $w$ belong to $J, m(e) \geq m$ and $r$ divides $r(e)$ or $v=y_{1} \cdots y_{t}$ and $w=y_{\sigma(1)} \cdots y_{\sigma(t)}$, with $t \geq n$, and $\sigma \in S_{t}$.

Note that $y_{1}, y_{2}, \ldots, y_{n}$ above need not be different.

Proof. Let $T^{*}(n, J, m, r)$ denote a set consisting of those equations $e$ of the form $v=w$ for which either $v$ and $w$ belong to $J, m(e) \geq m$, and $r$ divides $r(e)$ or $v=y_{1} \cdots y_{t}$, and $w=y_{\sigma(1)} \cdots y_{\sigma(t)}$, where $t \geq n, \sigma \in S_{t}$. It is easy to see that $T^{*}(n, J, m, r)$ is a theory. We show that $T^{*}(n, J, m, r)=T(n, J, m, r)$. Obviously, $T^{*}(n, J, m, r) \in S(n, J, m, r)$. We have to show that $T^{*}(n, J, m, r)$ is the least element in this class. Since $n\left(T^{*}(n, J, m, r)\right)=n$, all the equations $y_{1} \cdots y_{t}=$ $y_{\sigma(1)} \cdots y_{\sigma(t)}$, where $t \geq n, \sigma \in S_{t}$ belong to $T^{*}(n, J, m, r)$. By Lemma 4.4, all equations $e$ of the form $v=w$ such that $v, w \in J, m(e) \geq m$ and $r$ divides $r(e)$ belong to $T^{*}(n, J, m, r)$. Hence $T^{*}(n, J, m, r)$ is the least element in $S(n, J, m, r)$ and $T^{*}(n, J, m, r)=T(n, J, m, r)$.

For a subset $M$ of bqo $\left(W, \leq_{n}\right)$, we define $C l_{n}(M)=M \cup\left\{y_{\sigma(1)} \cdots y_{\sigma(k)}\right.$; $\left.y_{1} \cdots y_{k} \in M, k \geq n, \sigma \in S_{k}\right\}$. By $\mathcal{L}_{S}(S P)$ we denote the set of the theories of the form $T(n, J, m, r)$.

Corollary 4.7. Let $T_{1}=T\left(n_{1}, J_{1}, m_{1}, r_{1}\right)$ and $T_{2}=T\left(n_{2}, J_{2}, m_{2}, r_{2}\right)$. Then the following hold:

(i) $T_{1} \supseteq T_{2}$ if and only if $n_{1} \leq n_{2}, J_{1} \supseteq J_{2}, m_{1} \leq m_{2}$, and $r_{1}$ divides $r_{2}$,

(ii) $T_{1} \vee T_{2}=T(n, J, m, r)$, where $n=\min \left(n_{1}, n_{2}\right), J=C l_{n}\left(J_{1} \cup J_{2}\right), m=$ $\min \left(m_{1}, m_{2}\right)$, and $r=\operatorname{gcd}\left(r_{1}, r_{2}\right)$, 
(iii) the theory $T\left(\max \left(n_{1}, n_{2}\right), J_{1} \cap J_{2}, \max \left(m_{1}, m_{2}\right), \operatorname{lcm}\left(r_{1}, r_{2}\right)\right)$ is the largest theory in a set $\mathcal{L}_{S}(S P)$ that is contained in the set $T_{1} \cap T_{2}$.

Proof. The statement (i) is an immediate consequence of Theorem 4.6. Using (i), we have directly that (iii) holds and that $T(n, J, m, r)$ contains the set $T_{1} \cup T_{2}$. Assume that an equation $v=w$ belongs to $T(n, J, m, r)$. Then, either $v=y_{1} \cdots y_{k}$ and $w=y_{\sigma(1)} \cdots y_{\sigma(k)}$ for some $k \geq n$, and $\sigma \in S_{k}$ or $v, w \in J, m(v=w) \geq m$, and $r$ divides $r(v=w)$.

In the first case the equation $v=w$ belongs to $T_{1} \cup T_{2}$. In the latter, we may assume that $J_{1}$ is not empty. Let $v=y_{1} \cdots y_{k}, w=z_{1} \cdots z_{l}$. If $J_{2}$ is not empty, then there are the permutations $\sigma_{1} \in S_{k}, \sigma_{2} \in S_{l}$ (if $k \leq n$, then $\sigma_{1}$ is trivial, and if $l \leq n$, then $\sigma_{2}$ is trivial), and the word $u \in J_{1} \cap J_{2}$ such that the equations $u=y_{\sigma_{1}(1)} \cdots y_{\sigma_{1}(k)}$ and $u=z_{\sigma_{2}(1)} \cdots z_{\sigma_{2}(l)}$ belong to $T_{1} \cap T_{2}$. Hence, the equation $v=w$ is a consequence of the set $T_{1} \cap T_{2}$. If $J_{2}$ is empty, then the argument is similar. The only difference is that the word $u$ belongs to $J_{1}$. This implies that every equation which belongs to $T(n, J, m, r)$ is a consequence of the set of equations $T_{1} \cup T_{2}$. This proves (ii).

An immediate consequence of Corollary 4.7 is the following.

Theorem 4.8. The set $\mathcal{L}_{S}(S P)$ forms a lattice with operations $\vee$ and $\wedge$. For $T_{1}=T\left(n_{1}, J_{1}, m_{1}, r_{1}\right), T_{2}=T\left(n_{2}, J_{2}, m_{2}, r_{2}\right)$, and $n=\min \left(n_{1}, n_{2}\right)$, we have the following:

(i) $T_{1} \vee T_{2}=T\left(n, C l_{n}\left(J_{1} \cup J_{2}\right), \min \left(m_{1}, m_{2}\right), \operatorname{gcd}\left(r_{1}, r_{2}\right)\right)$,

(ii) $T_{1} \wedge T_{2}=T\left(\max \left(n_{1}, n_{2}\right), J_{1} \cap J_{2}, \max \left(m_{1}, m_{2}\right), \operatorname{lcm}\left(r_{1}, r_{2}\right)\right)$.

Moreover, $\mathcal{L}_{S}(S P)$ is $\vee$-subsemilattice of $\mathcal{L}(S P)$.

Since sometimes it may happen that $T_{1} \wedge T_{2} \notin \mathcal{L}_{S}(S P)$, the lattice $\mathcal{L}_{S}(S P)$ is not a sublattice of $\mathcal{L}(S P)$.

We are going to prove that the sets $S(n, J, m, r)$ are intervals in $\mathcal{L}(S P)$. We need the following lemma.

Lemma 4.9. Let $T_{1} \in S\left(n_{1}, J_{1}, m_{1}, r_{1}\right)$ and $T_{2} \in S\left(n_{2}, J_{2}, m_{2}, r_{2}\right)$. Then the following hold:

(i) $T_{1} \wedge T_{2} \in S\left(\max \left(n_{1}, n_{2}\right), J_{1} \cap J_{2}, \max \left(m_{1}, m_{2}\right), \operatorname{lcm}\left(r_{1}, r_{2}\right)\right)$,

(ii) $T_{1} \vee T_{2} \in S\left(n, J, \min \left(m_{1}, m_{2}\right), \operatorname{gcd}\left(r_{1}, r_{2}\right)\right)$, for some $n \leq \min \left(n_{1}, n_{2}\right)$ and $J \supseteq J_{1} \cup J_{2}$.

It may happen that $J \neq J_{1} \cup J_{2}$ (see [13] when $T_{1}, T_{2} \in \mathcal{L}(C o m)$ ). Similarly, there are examples that $n \neq \min \left(n_{1}, n_{2}\right)$. Consequently, $\equiv_{S}$ is not a congruence relation.

Proof. Since $T_{1} \wedge T_{2}=T_{1} \cap T_{2}$ and $T_{1} \vee T_{2} \supseteq T_{1} \cup T_{2}$, we immediately have suitable conditions on $n\left(T_{1} \wedge T_{2}\right), n\left(T_{1} \vee T_{2}\right), J\left(T_{1} \wedge T_{2}\right)$ and $J\left(T_{1} \vee T_{2}\right)$. It is also obvious that $m\left(T_{1} \cap T_{2}\right)=\max \left(m_{1}, m_{2}\right), r\left(T_{1} \cap T_{2}\right)=\operatorname{lcm}\left(r_{1}, r_{2}\right)$. Since $m\left(T_{1} \cup T_{2}\right)=\min \left(m_{1}, m_{2}\right)$, we have $m\left(T_{1} \vee T_{2}\right) \leq \min \left(m_{1}, m_{2}\right)$. Assume that $e \in T_{1} \vee T_{2}$ satisfies $m(e)=m\left(T_{1} \vee T_{2}\right)$. Then $e$ has a form $v_{1}=v_{k}$ and is obtained by transitivity from the equations $v_{i}=v_{i+1} ; i \in\{1, \ldots, k-1\}$. All the equations $v_{i}=v_{i+1}$ belong either to $T_{1}$ or to $T_{2}$. Let $x$ be a variable that belongs to $\operatorname{supp}\left(v_{1}\right) \cup \operatorname{supp}\left(v_{k}\right)$, and let $\left|v_{1}\right|_{x} \neq\left|v_{k}\right|_{x}$. Then there is $i \in\{1, \ldots, k-1\}$ such that $\left|v_{i}\right|_{x} \neq\left|v_{i+1}\right|_{x}$. This implies that $\min \left(m_{1}, m_{2}\right) \leq m\left(T_{1} \vee T_{2}\right)$, and we 
have the equality $m\left(T_{1} \vee T_{2}\right)=\min \left(m_{1}, m_{2}\right)$. Similarly, one can prove the equality $r\left(T_{1} \vee T_{2}\right)=\operatorname{lcm}\left(r_{1}, r_{2}\right)$.

Corollary 4.10. Every equivalence class $S(n, J, m, r)$ is an interval in $\mathcal{L}(S P)$.

\section{The Lattice $\mathcal{L}(S P)$}

Now we supplement the objects $n, J, m, r$ by an equivalence relation $\pi$ on a set $\mathcal{W} \backslash J$. Using these five objects, we describe every single theory in $\mathcal{L}(S P)$. The equivalence relation $\pi$ will play the same role as the similar equivalence relation that we define in section 2.2. The equivalence relation $\pi$ will be called a remainder of the theory $T$.

5.1. Remainders. Before we describe the structure of the lattice $\mathcal{L}(S P)$, we give some description of the structure of remainders. First, we prove the following.

Lemma 5.1. Let $v=w$ be the equation that belongs to $T$. If either

(a) $\operatorname{supp}(v) \neq \operatorname{supp}(w)$ (irregular equations)

$$
\text { or }
$$

(b) $v<_{n} w,(S$-equations),

then both $v$ and $w$ belong to $J(T)$.

Proof. We start with case (a). We assume, without loss of generality, that $x \in$ $\operatorname{supp}(v) \backslash \operatorname{supp}(w)$. Since $m(T)=0$, we know that $J(T)$ is not empty. Hence, there is a number $t$ such that $x^{t} \in J(T)$. Let $v^{\prime}$ denote the word obtained from $v$ by substituting in $v$ the variable $x$ by $z^{t}$, where $z \notin \operatorname{supp}(v) \cup \operatorname{supp}(w)$. It is obvious that $v^{\prime} \in J(T)$ and the equation $v^{\prime}=w$ belongs to $T$. It follows that $w \in J(T)$. Consequently, $v \in J(T)$. This completes the proof in case (a).

Now consider case (b). In view of (a), we may assume that $\operatorname{supp}(v)=\operatorname{supp}(w)=$ $\left\{x_{1}, \ldots, x_{k}\right\}$. Hence, we have, immediately, that $|v|<|w|$. Moreover, there is an operation $f \in \mathcal{O}$ such that either $f(v)=w$ or $\left(|f(v)|_{x_{1}}, \ldots,|f(v)|_{x_{k}}\right) \equiv_{K}$ $\left(|w|_{x_{1}}, \ldots,|w|_{x_{k}}\right)$. It follows that $\left|f^{t}(v)\right|<\left|f^{t+1}(v)\right|$, for every natural number $t$. Consequently, $\left|f^{n}(v)\right| \geq n$. Thus, the equation $f^{n}(v)=f^{n+1}(v)$ belongs to $T_{n}$. By Remark 2.9, $f^{n}(v) \in J_{n}(T) \subseteq J(T)$. By transitivity of equality, the equation $v=f^{n}(v)$ belongs to $T$. This implies that $v \in J(T)$ and $w \in J(T)$, and completes the proof of the lemma.

Let us define $\pi(T)$ to be the set

$$
(T \backslash T(n(T), J(T), m(T), r(T))) \cup\{v=v: v \in \mathcal{W} \backslash J(T)\} .
$$

Thus, $T=T(n(T), J(T), m(T), r(T)) \cup \pi(T)$. As in the commutative case, $\pi$ is a relatively small part of $T$, and similarly, we call it a remainder of the type $(n, J, m, r)$. For an equation $e=(v, w) \in T$, either $v, w \in J(T)$ and $e$ satisfies the conditions $(\mathrm{J} 1)$ and $(\mathrm{J} 2)$ or $v, w \notin J(T)$ and $e \in \pi(J)$. We establish properties that are satisfied by an equation $(v, w) \in \pi(T)$.

By Lemma 5.1, $\pi(T)$ can be viewed as an equivalence relation on the set $\mathcal{W} \backslash J(T)$.

Lemma 5.2. Let $T \in \mathcal{L}(S P)$ and $(v, w) \in \pi(T)$. Then the following conditions hold:

(П1) $\operatorname{supp}(v)=\operatorname{supp}(w)$,

(П2) conditions (J1) and (J2) hold,

(П3) for every $f \in \mathcal{O}$, either $(f(v), f(w)) \in \pi(T)$ or $f(v), f(w) \in J(T)$. 
Proof. The condition (П1) is an immediate consequence of Lemma 5.1. The condition (П2) holds for every equation $e \in T$. The condition (П3) is an immediate consequence of the fact that $T=T(n(T), J(T), m(T), r(T)) \cup \pi(T)$.

As a consequence of (П3) we have additional conditions.

Corollary 5.3. For $T, v, w$ as above, we have:

- $(\sigma(v), \sigma(w)) \in \pi(T)$, for every $\sigma \in P$,

- if for some $x \in \operatorname{supp}(v)$ we have $|v|_{x} \neq|w|_{x}, 2|v|_{x} \geq n(T)$ and $2|w|_{x} \geq n$, then $\left\{v t^{s}, t^{s} v, w t^{s}, t^{s} w\right\} \subset J(T)$, where $s=\min \left(|v|_{x},|w|_{x}\right)$.

Proof. Since $v, w \notin J, \sigma(v) \equiv_{n} v$, and $\sigma(w) \equiv_{n} w$, we have $\sigma(v), \sigma(w) \notin J$. Therefore, by $(\Pi 3),(\sigma(v), \sigma(w)) \in \pi$. Moreover, since $(v, w) \in T$, for substitutions we have $\left(t_{x z}(v), t_{x z}(w)\right) \in T$. Since $2|v|_{x} \geq n(T)$ and $2|w|_{x} \geq n(T)$, the following equations hold: $t_{x z}(v)=v z^{|v|_{x}}=z^{|v|_{x}} v$ and $t_{x v}(w)=w z^{|w|_{x}}=z^{|w|_{x}} w$. Using those properties, the transitivity of the equality, and the fact that $\left(v z^{s}, w z^{s}\right) \in T$ and $\left(z^{s} v, z^{s} w\right) \in T$, we have the second statement.

One may observe that, in fact, (П1) is also a consequence of (П3). However, (П1) is so important that it is worth mentioning it as a separate condition.

As in [4, we describe more precisely the structure of equivalence blocks of equivalence relations $\pi$. By Lemma 5.1 two words that belong to a block B have to be either incomparable or equivalent in the bqo $\left(\mathcal{W}, \leq_{n}\right)$. By Corollary 5.3 , the block $B$ is also the block for the symmetric group $S_{k}$ under its action on the words with support of cardinality $k$. Consequently, we have an induced action of $S_{k}$ on the set of blocks that consists of the words with the support of cardinality $k$. By $G_{B}$, as in [4], we denote the setwise stabilizer of $B$ in $S_{k}$ under this action. Since the block $B$ consists of the words that are either incomparable or equivalent, we may choose a maximal antichain $A \subseteq B$ such that, for every $v \in B$, there is $w \in A$ and $\sigma \in S_{k}$ with $\sigma(w)=v$.

It is clear that the block $B$ is determined by the antichain $A$ and the group $G_{B}$ as follows.

Lemma 5.4. For every block $B$ of a remainder $\pi$, if $A$ is a maximal antichain contained in $B$, then $B=G_{B} A=\left\{g(v): g \in G_{B}, v \in A\right\}$.

It is clear that if elements of $A$ have the support of cardinality $k$, then the group $G_{B}$ contains all point stabilizers of elements of $A$ in a group $S_{k}$. In fact, $G_{B}$ contains point stabilizers of all elements of $B$. We prove additional conditions that every remainder has to satisfy.

Lemma 5.5. Let $\pi$ be a remainder of type $(n, J, m, r)$ and $B$ be a block of $\pi$ that contains a word $x_{1} \cdots x_{k}$, with $k<n$. Then the following holds:

(1) $B$ consists of the words of the form $x_{\sigma(1)} \cdots x_{\sigma(k)}$, where $\sigma \in S_{k}$.

(2) $G_{B} \neq S_{k}$.

Proof. We prove (2). Since, $\pi=T \backslash T(n(T), J(T), m(T), r(T))$ for some $T$ with $n(T)=n, J(T)=J, m(T)=m$ and $r(T)=r$, there is $\sigma \in S_{n}$ such that $\left(x_{1} \cdots x_{k}, x_{\sigma(1)} \cdots x_{\sigma(k)}\right) \notin T \supseteq \pi$.

Now we prove (1). We assume, to the contrary, that there exists a word $w$ in $B$ that is not in the form $x_{\sigma(1)} \cdots x_{\sigma(k)}$. Then, by (П1), $|w|>k$. Using substitutions, identification variables and transitivity, it is easy to find a word $v \in B$ such that 
$|v| \geq n$. By (П1) $\operatorname{supp}(v)=\operatorname{supp}(w)$. Thus, we may choose $v$ of the form $x_{1}^{i_{1}} \cdots x_{k}^{i_{k}}$, where at least one $i_{j}$ is greater than 1. But, obviously, $x_{1} \cdots x_{k}<v$. This is in contradiction with Lemma 5.1(b).

For a fixed remainder $\pi$, we may define a quasi-ordering on a set of equivalence blocks of $\pi$. We define $B_{1} \leq_{\pi} B_{2}$ if and only if there is $f \in \mathcal{O}$ such that $f\left(B_{1}\right) \subseteq B_{2}$. By (П3), this definition is equivalent to the condition that there is $b \in B_{1}$ and $f \in \mathcal{O}$ such that $f(b) \in B_{2}$.

Since $\left(W, \leq_{n}\right)$ is bqo, using Fact 2.4 we have the following.

Corollary 5.6. The quasi-ordering $\left((\mathcal{W} \backslash J)_{/ \pi}, \leq_{\pi}\right)$ is bqo.

In the same way as in [4, Lemma 1.2], we obtain the following.

Lemma 5.7. Let $B, C \in \pi$. Then $B$ and $C$ are equivalent if and only if $p(B)=C$ for some $p \in P$.

Fix a positive integer $n$. Since there are only finitely many words $w$ such that $|w|<n$ and $\operatorname{supp}(w)=\left\{x_{1}, \ldots, x_{k}\right\}$, we have only finite many blocks $B \in \pi$ such that there is $w \in B$ with $|w|<n$. Hence, by Remark 2.10 we have the following.

Corollary 5.8. For every remainder $\pi$ there is a finite set $\bar{\pi}$ of blocks of $\pi$ such that every block $C \in \pi$ has a form $p l^{*} r^{*}(B)$ for some $B \in \bar{\pi}, p \in P$ and operations $l^{*}, r^{*}$ of the form $r^{*}=\left(r_{y_{1}}\right)^{t_{1}} \cdots\left(r_{y_{k}}\right)^{t_{k}}$ and $l^{*}=\left(l_{z_{1}}\right)^{s_{1}} \cdots\left(l_{z_{j}}\right)^{s_{j}}$.

5.2. Theories of $\mathcal{L}(S P)$. The following theorem describes every theory of $\mathcal{L}(S P)$ using the objects $n, J, m, r, \pi$.

Theorem 5.9. Given integers $m \geq 0, r>0, n \geq 2$, and an order filter $J$ such that $m(J)=m$ and $x_{1} \cdots x_{n-1} \notin J$, let $\pi$ be an equivalence relation on the set $\mathcal{W} \backslash J$ satisfying conditions (П1),(П2) and (П3) and such that for the block $B$ with $x_{1} \cdots x_{n-1} \in B$, we have $G_{B} \neq S_{n-1}$. Let $T(n, J, m, r, \pi)=T(n, J, m, r) \cup \pi$. Then the following hold:

(i) $T(n, J, m, r, \pi) \in \mathcal{L}(S P)$,

(ii) $T(n, J, m, r, \pi) \in S(n, J, m, r)$,

(iii) $(v, w) \in T(n, J, m, r, \pi)$ if and only if either both $v, w \in J$ and satisfy conditions $(\mathrm{J} 1)$ and $(\mathrm{J} 2)$ or both $v, w \notin J$ and $(v, w) \in \pi$.

Conversely, every $T \in \mathcal{L}(S P)$ other than $\mathcal{I}$ is of this form.

Proof. We prove (i). We have to show that the consequences of the equations that belong to $T$, themselves, belong to $T$. For $(v, w) \in T$ and $(w, u) \in T$ we have to show that

(a) $(r(v), r(w)) \in T$,

(b) $(l(v), l(w)) \in T$,

(c) $\left(g_{x_{i} x_{j}}(v), g_{x_{i} x_{j}}(w)\right) \in T$,

(d) $\left(t_{x_{i} y}(v), t_{x_{i} y}(w)\right) \in T$,

(e) $(p(v), p(w)) \in T$, and

(f) $(v, u) \in T$.

Since $T(n, J, m, r) \in \mathcal{L}(S P)$ and by the definition, if $\left(v^{\prime}, w^{\prime}\right) \in T$, then either $\left(v^{\prime}, w^{\prime}\right) \in T(n, J, m, r)$ and $v^{\prime}, w^{\prime} \in J$ or $\left(v^{\prime}, w^{\prime}\right) \in \pi$ and $v^{\prime}, w^{\prime} \notin J$, it is enough to check these conditions with assumptions $(v, w) \in \pi$ and $(w, u) \in \pi$.

By Corollary [5.3, (e) holds. To check (a)-(d), let $f$ be one of the operations $r, l, g_{x_{i} x_{j}}, t_{x_{i}, y}$. By $(\Pi 3)$ either $(f(v), f(w)) \in \pi$ and we are done or $f(v), f(w) \in J$. 
Moreover, by (П2) the conditions (J1) and (J2) hold for $(v, w)$. We show that they hold for $(f(v), f(w))$. This is clear for $f \in\left\{r, l, t_{x_{i} y}\right\}$. Let $f=g_{x_{i}, x_{j}}$. Assume $|f(v)|_{x_{h}} \neq|f(w)|_{x_{h}}$. If $h \neq i$, then $|f(v)|_{x_{h}}=|v|_{x_{h}}$ and $|f(w)|_{x_{h}}=|w|_{x_{h}}$. We have $|v|_{x_{h}} \geq m,|w|_{x_{h}} \geq m$, and $r$ divides $|v|_{x_{h}}-|w|_{x_{h}}$. If $h=i$, then $|f(v)|_{x_{h}}=$ $|v|_{x_{i}}+|v|_{x_{j}}$ and $|f(w)|_{x_{h}}=|w|_{x_{i}}+|w|_{x_{j}}$. Obviously, $r$ divides $|f(v)|_{x_{h}}-|f(w)|_{x_{h}}$. Moreover, either $|v|_{x_{i}} \neq|w|_{x_{i}},|v|_{x_{i}} \geq m$, and $|w|_{x_{i}} \geq m$ or $|v|_{x_{j}} \neq|w|_{x_{j}},|v|_{x_{j}} \geq m$, and $|w|_{x_{j}} \geq m$. Therefore, (J1) and (J2) hold for $(f(v), f(w))$. Thus, $(f(v), f(w)) \in$ $T$. This proves (i).

Since $T(n, J, m, r) \subseteq T$ and for the block $B$ with $x_{1} \cdots x_{n} \in B$ we have $G_{B} \neq S_{n}$, it is clear that $n(T)=n$. Moreover, $J(T)=J$, since in addition we have (П3). Finally, $m(T)=m$ and $r(T)=r$, since in addition (J1) and (J2) hold for every equation in $T$. This proves (ii). Now (iii) is obvious.

The converse holds by Lemma 5.2

Since every order filter in the set $\left(\mathcal{W}, \leq_{n}\right)$ is finitely generated, using Corollary 5.8, we obtain the following well-known fact that we will need later.

Theorem 5.10. Every equational theory of strongly permutative semigroups is finitely generated.

The above is an easy consequence of the results of Perkins [18] and Putcha-Yaqub 20 .

\section{Structure of $\mathcal{L}(S P)$}

In this section, we describe the structure of $\mathcal{L}(S P)$ as a lattice and as an ordering.

6.1. Inclusion and the operations $\vee, \wedge$. For two theories $T_{1}, T_{2} \in \mathcal{L}(S P)$, we describe conditions under which $T_{1} \subseteq T_{2}$. Moreover, we determine theories $T_{1} \wedge T_{2}$ and $T_{1} \vee T_{2}$.

Theorem 6.1. Let $T_{1}=T\left(n_{1}, J_{1}, m_{1}, r_{1}, \pi_{1}\right)$ and $T_{2}=T\left(n_{2}, J_{2}, m_{2}, r_{2}, \pi_{2}\right)$. Then $T_{1} \supseteq T_{2}$ if and only if $n_{1} \leq n_{2}, J_{1} \supseteq J_{2}, m_{1} \leq m_{2}, r_{1}$ divides $r_{2}$, and $\pi_{1} \cup J_{1}^{2} \supseteq \pi_{2}$.

Proof. The "only if" part is obvious. For the "if" part, since $n_{1} \leq n_{2}$, it is enough to show that if $(v, w) \in \pi_{2} \subseteq \pi_{1} \cup J_{1}^{2}$, then $(v, w) \in E_{1}$. If $(v, w) \in \pi_{1}$, then this is obvious. If $(v, w) \in J_{1}^{2}$, then, since the equation $(v, w)$ satisfies condition $\Pi_{2}$ with $m_{2}$ and $r_{2}$, it satisfies $\Pi_{2}$ with $m_{1}$ and $r_{1}$. Therefore, $(v, w) \in T\left(n_{1}, J_{1}, m_{1}, r_{1}\right) \subseteq$ $T_{1}$, as required.

For an order filter $J, m \geq 0$, and $r>0$, by $T(J, m, r)$ we denote a set of those $(v, w)$ with $v, w \in J$ that satisfy conditions (J1) and (J2) with parameters $m$ and $r$.

Theorem 6.2. Let $T_{1}=T\left(n_{1}, J_{1}, m_{1}, r_{1}, \pi_{1}\right)$ and $T_{2}=T\left(n_{2}, J_{2}, m_{2}, r_{2}, \pi_{2}\right)$. Then, $T_{1} \wedge T_{2}=T_{1} \cap T_{2}=T\left(\max \left(n_{1}, n_{2}\right), J_{1} \cap J_{2}, \max \left(m_{1}, m_{2}\right), \operatorname{lcm}\left(r_{1}, r_{2}\right), \pi\right)$, where $\pi=\left(\pi_{1} \cap \pi_{2}\right) \cup\left(\pi_{1} \cap T\left(J_{2}, m_{2}, r_{2}\right)\right) \cup\left(\pi_{2} \cap T\left(J_{1}, m_{1}, r_{1}\right)\right)$.

Proof. The equality $n\left(T_{1} \cap T_{2}\right)=\max \left(n_{1}, n_{2}\right)$ is obvious. The rest is a consequence of the fact that $\left(T\left(J_{1}, m_{1}, r_{1}\right) \cup \pi_{1}\right) \cap\left(T\left(J_{2}, m_{2}, r_{2}\right) \cup \pi_{2}\right)=\left(T\left(J_{1}, m_{1}, r_{1}\right) \cap\right.$ $\left.T\left(J_{2}, m_{2}, r_{2}\right)\right) \cup\left(T\left(J_{1}, m_{1}, r_{1}\right) \cap \pi_{2}\right) \cup\left(T\left(J_{2}, m_{2}, r_{2}\right) \cap \pi_{1}\right) \cup\left(\pi_{1} \cap \pi_{2}\right)=T\left(J_{1} \cap\right.$ $\left.J_{2}, \max \left(m_{1}, m_{2}\right), \operatorname{lcd}\left(r_{1}, r_{2}\right)\right) \cup\left(T\left(J_{1}, m_{1}, r_{1}\right) \cap \pi_{2}\right) \cup\left(T\left(J_{2}, m_{2}, r_{2}\right) \cap \pi_{1}\right) \cup\left(\pi_{1} \cap\right.$ $\left.\pi_{2}\right)$. 
Before we formulate a similar theorem for the operation $\vee$, we give some definitions. Let $T_{1}=T\left(n_{1}, J_{1}, m_{1}, r_{1}, \pi_{1}\right)$ and $T_{2}=T\left(n_{2}, J_{2}, m_{2}, r_{2}, \pi_{2}\right)$. We denote $G_{n}\left(T_{i}\right)=S_{n}$ for $n \geq n_{i}$ and $G_{n}\left(T_{i}\right)=G_{B}$ if $n<n_{i}$, and $x_{1} \cdots x_{n}$ belongs to the class $B$ in $\pi_{i}$. Now, we define, inductively, $G_{n}\left(T_{1} \cup T_{2}\right)$ :

$$
\begin{aligned}
& G_{2}\left(T_{1} \cup T_{2}\right)=\left\langle G_{2}\left(T_{1}\right), G_{2}\left(T_{2}\right)\right\rangle, \\
G_{k+1}\left(T_{1} \cup T_{2}\right)= & \left\langle G_{k+1}\left(T_{1}\right), G_{k+1}\left(T_{2}\right), G_{k}\left(T_{1}\right) \times S_{1}, G_{k}\left(T_{2}\right) \times S_{1},\right. \\
& \left.S_{1} \times G_{k}\left(T_{1}\right), S_{1} \times G_{k}\left(T_{2}\right), G_{k}^{i}\left(T_{1}\right), G_{k}^{i}\left(T_{2}\right)\right\rangle,
\end{aligned}
$$

where $i \in\{1, \ldots, k\}$ and $G_{k}^{i}\left(T_{j}\right)$ is a subgroup of $S_{k+1}$ generated by permutations $\sigma^{i}: \sigma \in G_{k}\left(T_{j}\right)$, and $\sigma^{i}$ rearranges the set $\{1, \ldots, k+1\}$ just as $\sigma$ rearranges the set $\{1, \ldots, k\}$ treating the pair $i, i+1$ as a single element. See [15] for details. In fact $G_{k}\left(T_{1} \cup T_{2}\right)$ is a minimal subgroup of $S_{k}$ generated by all permutations of this form arising from the groups $\left\langle G_{l}\left(T_{1}\right), G_{l}\left(T_{2}\right)\right\rangle$ for all $l \leq k$.

By $n\left(T_{1} \cup T_{2}\right)$, we denote a minimal $n$ such that $S_{n}=G_{n}\left(T_{1} \cup T_{2}\right)$. Obviously, $n\left(T_{1} \vee T_{2}\right) \leq \min \left(n_{1}, n_{2}\right)$. We also consider the equivalence relation $R$ which is the equivalence closure of the relation $J_{1}^{2} \cup J_{2}^{2} \cup \pi_{1} \cup \pi_{2}$. By $J\left(T_{1} \cup T_{2}\right)$ we denote the equivalence block in $R$ which contains the set $J_{1} \cup J_{2}$. Moreover, by $\pi\left(T_{1} \cup T_{2}\right)$ we denote the equivalence relation $R \backslash\left(J\left(T_{1} \cup T_{2}\right)\right)^{2}$ on the set $\mathcal{W} \backslash J\left(T_{1} \cup T_{2}\right)$.

Theorem 6.3. Let $T_{1}=T\left(n_{1}, J_{1}, m_{1}, r_{1}, \pi_{1}\right)$ and $T_{2}=T\left(n_{2}, J_{2}, m_{2}, r_{2}, \pi_{2}\right)$. Then $T_{1} \vee T_{2}=T\left(n\left(T_{1} \cup T_{2}\right), J\left(T_{1} \cup T_{2}\right), \min \left(m_{1}, m_{2}\right), \operatorname{gcd}\left(r_{1}, r_{2}\right), \pi\left(T_{1} \cup T_{2}\right)\right)$.

Proof. The equality $n\left(T_{1} \vee T_{2}\right)=n\left(T_{1} \cup T_{2}\right)$ is an immediate consequence of the definition of $n\left(T_{1} \cup T_{2}\right)$, Lemma $5.5(1)$, and the fact that if the equation $x_{1} \cdots x_{k}=x_{\sigma(1)} \cdots x_{\sigma(k)}$ with $k<\min \left(n_{1}, n_{2}\right)$ is a consequence of the set of equations $\left\{e_{1}, \ldots, e_{j}\right\}$, then every equation $e_{l}$ has a form $p_{1}\left(x_{1} \cdots x_{i}\right)=p_{2}\left(x_{1} \cdots x_{i}\right)$ for some $i \leq k$, and permutations $p_{1}, p_{2} \in P$. (Otherwise, there is $x_{1} \cdots x_{i} \in J_{l}$ for some $i \leq k$ and $l \in\{1,2\}$. In this situation $n\left(T_{l}\right) \leq i$, which contradicts the fact that $i \leq k<n\left(T_{l}\right)$.)

The equalities $J\left(T_{1} \vee T_{2}\right)=J\left(T_{1} \cup T_{2}\right)$ and $\pi\left(T_{1} \vee T_{2}\right)=\pi\left(T_{1} \cup T_{2}\right)$ are consequences of the fact that taking transitivity of the equations is the same as taking the transitive closure of the relation on equations and the fact that the relation $J_{1}^{2} \cup J_{2}^{2} \cup \pi_{1} \cup \pi_{2}$ is symmetric and reflective and that these properties are preserved by transitive closure. The equality $m\left(T_{1} \vee T_{2}\right)=\min \left(m_{1}, m_{2}\right)$ holds by the fact that we have $m(w, v) \geq \min (m(w, u), m(u, v))$ for every $w, v, u \in W$. Finally, we prove the equality $r\left(T_{1} \vee T_{2}\right)=\operatorname{gcd}\left(r_{1}, r_{2}\right)$.

The inequality $r\left(T_{1} \vee T_{2}\right) \geq \operatorname{gcd}\left(r_{1}, r_{2}\right)$ holds by the fact that we have $m(w, v) \geq$ $\operatorname{gcd}(m(w, u), m(u, v))$ for every $w, v, u \in W$. If either $r_{1}=\infty$ or $r_{2}=\infty$, then the opposite inequality is obvious. Assume that both $r_{1}<\infty$ and $r_{2}<\infty$ hold. Then, there is $k$ such that the equation $x^{k}=x^{k+r_{1}}$ belongs to $T_{1}$ and the equation $x_{k}=x^{k+r_{1}}$ belongs to $T_{2}$. It is easy to see that the equation $x^{k}=x^{k+\operatorname{gcd}\left(r_{1}, r_{2}\right)}$ belongs to $T_{1} \vee T_{2}$. This proves the second inequality and completes the proof.

6.2. Covering relation. In this section we describe, in terms of the objects $n, J$, $m, r, \pi$, the covering relation in the lattice $\mathcal{L}(S P)$. Let $T \in \mathcal{L}(S P)$. We say that $T_{1}$ is a cover of $T$ in $\mathcal{L}(S P)$ if $T_{1}>T$ and there is no $T_{2}$ such that $T_{1}>T_{2}>T$. In this case, we also say that $T$ is a dual cover of $T_{1}$.

For an antichain $A$ of minimal words in $J$ that have the same $n$ variables, we say that $A$ is an $(n, m, r)$-separable form $J$ if for every $v, w \in A$ there is $p \in S_{n}$ such that the conditions (J1) and (J2) are satisfied for the pair $(v, p(w))$. Then, by $\pi_{A}$, we 
denote the set of all those pairs $(v, w)$ such that $p(v), q(w) \in A$ for some $p, q \in P$, and the conditions (J1) and (J2) are satisfied. Let $\left\{x_{1} \cdots x_{n}\right\}$ be a one-element antichain.

Theorem 6.4. Let $T_{1}=T\left(n_{1}, J_{1}, m_{1}, r_{1}, \pi_{1}\right)$ and $T_{2}=T\left(n_{2}, J_{2}, m_{2}, r_{2}, \pi_{2}\right)$ be equational theories of strongly permutative semigroups. Then $T_{2}$ is a dual cover of $T_{1}$ if and only if one of the following holds:

(1) $n_{2}=n_{1}+1, J_{2}=J_{1}, m_{2}=m_{1}, r_{2}=r_{1}$, and

$$
\pi_{2}=\left(\pi_{1} \backslash\left\{p S_{n}\left\{\left(x_{1} \cdots x_{n}\right)\right\}: p \in P\right\}\right) \cup\left\{p G\left\{\left(x_{1} \cdots x_{n}\right)\right\}: p \in P\right\},
$$

where $G$ is a co-atom in $S_{n}$.

(2) $n_{2}=n_{1}, J_{2}=J_{1} \backslash \bigcup_{p \in P} p A, m_{2}=m_{1}, r_{2}=r_{1}$, and $\pi_{2}=\pi_{1} \cup \pi_{A}$, where $A \subset J_{1}$ is an $\left(n_{1}, m_{1}, r_{1}\right)$-separable antichain.

(3) $n_{2}=n_{1}, J_{2}=J_{1}, m_{2}=m_{1}+1, r_{2}=r_{1}$, and $\pi_{2}=\pi_{1}$.

(4) $n_{2}=n_{1}, J_{2}=J_{1}, m_{2}=m_{1}, r_{2}=r_{1} q$, and $\pi_{2}=\pi_{1}$, where $q$ is a prime.

(5) $n_{2}=n_{1}, J_{2}=J_{1}, m_{2}=m_{1}, r_{2}=r_{1}$, and either

(a) $\pi_{2}=\left(\pi_{1} \backslash\{p(G A): p \in P\}\right) \cup\{p(H A): p \in P\}$ or

(b) $\pi_{2}=\left(\pi_{1} \backslash\{p(G A): p \in P\}\right) \cup\left\{p\left(G A_{1}\right) \cup p\left(G A_{2}\right): p \in P\right\}$, where $G, H \subseteq S_{n}, H$ is a dual cover of $G, A, A_{1}, A_{2}$ are antichains such that $A=A_{1} \cup A_{2}, A_{1} \cap A_{2}=\emptyset$, and $n$ is a number of variables of elements of $A$.

Proof. We prove the "if" part. It is obvious that in every case $T_{2}<T_{1}$. Let $T=T(n, J, m, r, \pi)$ be such that $T_{2} \leq T \leq T_{1}$. We must show that either $T=T_{1}$ or $T=T_{2}$. This is obvious in cases (3) and (4).

In case (1), we have $J=J_{1}=J_{2}, m=m_{1}=m_{2}, r=r_{1}=r_{2}$. If $n=n_{1}$, then the set $\left\{p\left(x_{1} \cdots x_{n}\right): p \in S_{n}\right\}$ has to be a subset of a block of $\pi_{1}$. Therefore, $T=T_{1}$. If $n=n_{2}$, then the set $\left\{p\left(x_{1} \cdots x_{n}\right): p \in S_{n}\right\}$ cannot be a subset of a block of $\pi_{1}$. This is possible only if $T=T_{2}$.

In case (2), we have $n=n_{1}=n_{2}, m=m_{1}=m_{2}, r=r_{1}=r_{2}$. Assume first that there exists $u \in A \backslash J$. Since $A$ is $\left(n_{1}, m_{1}, r_{1}\right)$-separable from $J_{1}$, for every pair $v, w \in A$ there is $p \in S_{n}$ such that $(v, p(w)) \in \pi_{A} \subseteq \pi_{2}$. Since $T \supseteq T_{2}$ and $u \notin J$, we have $A \cap J=\emptyset$. Therefore $J=J_{2}$. Then it is easy to see that $\pi=\pi_{2}$. Hence, $T=T_{2}$. If $J=J_{1}$, then it is clear that $\pi=\pi_{1}$, and hence $T=T_{1}$.

In case (5), we have $n=n_{1}=n_{2}, J=J_{1}=J_{2}, m=m_{1}=m_{2}, r=r_{1}=r_{2}$. In both subcases, it is clear that if $\pi>\pi_{2}$, then $\pi=\pi_{1}$, and $T=T_{1}$. Otherwise, $T=T_{2}$.

It remains to show the "only if" part. First we show that if either $m_{2} \neq m_{1}$ or $r_{2} \neq r_{1}$, then we have cases (3) or (4), respectively.

Indeed, if $m_{2}>m_{1}$, then $T=T\left(n_{2}, J_{2}, m_{2}-1, r_{2}, \pi_{2}\right)$ is a well-defined theory and $E_{2}<E \leq E_{1}$. Hence, $E=E_{1}$, and we have case (3).

If $r_{2}<r_{1}$, then, similar to the case above, a set $T=T\left(n_{2}, J_{2}, m_{2}, r_{2} / q, \pi_{2}\right)$ is a well-defined theory such that $T_{1}<T \leq T_{2}$ (where $q$ is a prime dividing $r_{1} / r_{2}$ ). Thus, $T=T_{1}$ and case (4) holds.

Now, suppose that $m_{2}=m_{1}, r_{2}=r_{1}$, and $n_{2}>n_{1}$. We show that this must be case (1). But before we do this, we need the following lemma.

Lemma 6.5. Under the condition above, $J_{2}=J_{1}$.

Proof. Assume to the contrary that $J_{1} \neq J_{2}$. First, we show that the word $x_{1} \cdots x_{n_{2}-1}$ does not belong to $J_{1}$. Otherwise, let $n$ be the smallest such that 
the word $x_{1} \cdots x_{n}$ belongs to $J_{1}$. The one-element set $\left\{x_{1} \cdots x_{n}\right\}$ is $\left(n_{1}, m_{1}, r_{1}\right)$ separable from $J_{1}$. Moreover, if $v \leq x_{1} \cdots x_{n}$, then $v=y_{1} \cdots y_{k}$, where $k \leq n$ and $y_{i} \neq y_{j}$ for every $i$ and $j$. Therefore, $T_{3}=T\left(n_{1}, J_{3}, m_{1}, r_{1}, \pi_{3}\right)$, where $J_{3}=J_{1} \backslash\left\{p\left\{x_{1} \cdots x_{n}\right\}: p \in P\right\}$ and $\pi_{3}=\pi_{1} \cup\left\{p S_{n}\left\{x_{1} \cdots x_{n}\right\}: p \in P\right\}$ is a well-defined theory and is a dual cover of $T_{1}$. Since $x_{1} \cdots x_{n} \notin J_{2}$, we have $T_{3} \geq T_{2}$. Hence, $T_{3}=T_{2}$. This contradicts the assumption $n_{1} \neq n_{2}$.

Now we consider a set $T_{4}=T\left(n_{2}, J_{1}, m_{2}, r_{2}, \pi_{4}\right)$, where $\pi_{4}=\pi_{2} \backslash J_{1}^{2}$. We show that $T_{4} \in \mathcal{L}(S P)$. Since $m_{2}=m_{1}$ and $x_{1} \cdots x_{n_{2}} \notin J_{1}$, conditions on $J_{1}$ are satisfied. We check conditions (П1) $-(\Pi 3)$. (П1) is obvious. Since $\pi_{4} \subseteq \pi_{2}$ and the parameters $m$ and $r$ are the same, (П2) holds for $\pi_{4}$. We check (П3). Let $f \in \mathcal{O}$. It is enough to show that if $(v, w) \in \pi_{4}$ and $f(v) \in J_{1}$, then $f(w) \in J_{1}$. It is clear by the fact that $T_{1}>T_{2}$ and $\pi_{4} \subseteq \pi_{2}$. Thus, $T_{4} \in \mathcal{L}(S P)$. It is clear that $T_{1} \geq T_{4} \geq T_{2}$. Since $n_{1}>n_{2}, T_{1}>T_{4}$. Hence, $T_{4}=T_{2}$. Thus, $J_{1}=J_{2}$. This completes the proof of the lemma.

Let $B \in \pi_{2}$ be a block containing the word $x_{1} \cdots x_{n_{1}}$. We consider an equivalence relation $\pi_{5}$ defined as follows: $\pi_{5}=\pi_{2} \cup\left\{C: C \in \pi_{1}, C>_{\pi} B\right\}$. It is easy to verify that $\pi_{5}$ is a remainder of the type $\left(n_{1}+1, J_{2}, m_{2}, r_{2}\right)$ and $\pi_{1} \supseteq \pi_{5} \supseteq \pi_{2}$. Let $T_{5}=T\left(n_{1}+1, J_{2}, m_{2}, r_{2}, \pi_{5}\right)$. Obviously, $T_{1} \geq T_{5} \geq T_{2}$ and $T_{5} \neq T_{1}$. Thus, $T_{5}=T_{2}$. Consequently, $n_{2}=n_{1}+1$. Moreover, $\pi_{2}$ and $\pi_{1}$ coincide on all blocks $\leq_{\pi}$ greater than $B$.

We consider an equivalence relation $\pi_{6}$ defined as follows:

$$
\pi_{6}=\left(\pi_{2} \backslash\{p B: p \in P\}\right) \cup\left\{p S_{n_{1}}\left\{x_{1} \cdots x_{n_{1}}\right\}: p \in P\right\} .
$$

It is easy to verify that $\pi_{6}$ is a remainder of the type $\left(n_{1}, J_{1}, m_{1}, r_{1}\right)$ and $\pi_{1} \supseteq$ $\pi_{6} \supseteq \pi_{2}$. Let $T_{6}=T\left(n_{1}, J_{1}, m_{1}, r_{1}, \pi_{6}\right)$. Obviously, $T_{1} \geq T_{6} \geq T_{2}$ and $T_{6} \neq T_{2}$. Thus, $T_{6}=T_{1}$. Consequently, $\pi_{2}$ coincides with $\pi_{1}$ on all blocks that are not $\leq_{\pi}$-equivalent to $B$.

Let $G$ be a maximal subgroup of $S_{n_{1}}$ such that $G_{B} \subseteq G$. We consider an equivalence relation $\pi_{7}$ defined as follows:

$$
\pi_{7}=\left(\pi_{2} \backslash\{p B: p \in P\}\right) \cup\left\{p G\left\{x_{1} \cdots x_{n_{1}}\right\}: p \in P\right\} .
$$

It is easy to verify that $\pi_{7}$ is a remainder of the type $\left(n_{2}, J_{2}, m_{2}, r_{2}\right)$ and $\pi_{1} \supseteq$ $\pi_{7} \supseteq \pi_{2}$. Let $T_{7}=T\left(n_{2}, J_{2}, m_{2}, r_{2}, \pi_{7}\right)$. Obviously, $T_{1}>T_{7} \geq T_{2}$. Thus, $T_{7}=T_{2}$. Consequently, $G=G_{B}$, and we have case (1).

Now suppose that $n_{2}=n_{1}, m_{2}=m_{1}, r_{2}=r_{1}$, and $J_{2} \neq J_{1}$. We show that this must be case (2). Let $\pi_{8}=\pi_{2} \backslash J_{1}^{2}$. It is easy to see that $\pi_{8}$ is a remainder of the type $\left(n_{1}, J_{1}, m_{1}, r_{1}\right)$. Let $T_{8}=T\left(n_{1}, J_{1}, m_{1}, r_{1}, \pi_{8}\right)$ and then, $T_{1} \geq T_{8}>T_{2}$. Thus, $T_{8}=T_{1}$. Therefore, $\pi_{1}=\pi_{2} \backslash J_{1}^{2}$.

Let $B \in \pi_{2}$ be a minimal block that is not a block in $\pi_{1}$. We consider an equivalence relation $\pi_{9}=\pi_{1} \cup\{p B: p \in P\}$. Let $J_{9}=J_{1} \backslash\{v$ : there is $p \in$ $P$ such that $p(v) \in B\}$. Since $J_{2} \subseteq J_{9} \subseteq J_{1}, m_{1}=m_{2}$, and $n_{1}=n_{2}$, the conditions on $J_{9}$ are satisfied. We prove that $\pi_{9}$ is a remainder of the type $\left(n_{1}, J_{1}, m_{1}, r_{1}\right)$. Conditions (П1) and (П2) are obvious. We check (П3). Assume $(v, w) \in \pi_{9}$ and $f \in \mathcal{O}$. Since $\pi_{9} \supseteq \pi_{2} \backslash J_{9}^{2}$, it is enough to show that if $f(v) \in J_{9}$, then $f(w) \in J_{9}$. If $f(v) \in J_{9}$, then $f(v) \in J_{1}$. Hence, $f(w) \in J_{1}$. Moreover, $f(v) \notin p B$ for any $p \in P$. Therefore, $f(w) \notin p B$ for any $p \in P$. Hence, $f(w) \in J_{9}$. Let $T_{9}=T\left(n_{1}, J_{9}, m_{1}, r_{1}, \pi_{9}\right)$. We have $T_{1}>T_{2} \geq T_{2}$. Hence, $T_{9}=T_{1}$. 
Let $A$ be an antichain in $B$. It is clear that all elements of $A$ are minimal in $J_{1}$. Since $n_{1}=n_{2}, m_{1}=m_{2}, r_{1}=r_{2}$, and $A$ is the subset of a block in $\pi_{2}, A$ is $\left(n_{1}, m_{1}, r_{1}\right)$-separable from $J_{1}$. Let $T_{10}=T\left(n_{2}, J_{2}, m_{2}, r_{2}, \pi_{10}\right)$, where $\pi_{10}=\pi_{1} \cup \pi_{A}$. Then $T_{10}$ is a dual cover of $T_{1}$. It is easy to see that $T_{10} \supseteq T_{2}$. Thus, $T_{10}=T_{2}$, and we have case $(2)$.

We show that the remaining cases $\left(n_{1}=n_{2}, J_{1}=J_{2}, m_{1}=m_{2}\right.$, and $\left.r_{1}=r_{2}\right)$ lead to case (5). The idea of the proof is the same as in [4, Section 3].

Let $B \in \pi_{1}$ be minimal such that $B$ is not a block in $\pi_{2}$. Let

$$
\pi_{11}=\left(\pi_{1} \backslash\{p B: p \in P\}\right) \cup\left\{p C: C \in \pi_{2}, C \subseteq B, p \in P\right\} .
$$

We leave it to the reader to check that $\pi_{11}$ is a remainder of the form $\left(n_{1}, J_{1}, m_{1}, r_{1}\right)$ (see [4, Lemma 3.2]). Since $\pi_{1} \supseteq \pi_{11} \supseteq \pi_{2}$ and $\pi_{11} \neq \pi_{1}$, we have $\pi_{11}=\pi_{2}$. Consequently, $\pi_{2}$ differs from $\pi_{1}$ only within the blocks that are equivalent to $B$. We show that this difference can be only of the kind given in $(5)(\mathrm{a})$ or $(5)(\mathrm{b})$.

Let $C \in \pi_{2}$ such that $C \subseteq B$. We define $C^{B}=G_{B} C$. Let

$$
\pi_{12}=\left(\pi_{2} \backslash\left\{p C: C \in \pi_{2}, C \subseteq B, p \in P\right\}\right) \cup\left\{p C^{B}: C \subseteq B, p \in P\right\} .
$$

Again, we leave it to the reader to check that $\pi_{12}$ is a remainder of the form $\left(n_{1}, J_{1}\right.$, $m_{1}, r_{1}$ ) (see [4, Lemma 3.4]). Since $\pi_{1} \supseteq \pi_{12} \supseteq \pi_{2}$, we have either $\pi_{12}=\pi_{1}$ or $\pi_{12}=\pi_{2}$. We show that these two possibilities lead to the possibilities (5)(a) and (5) (b), respectively.

First, assume that $\pi_{12}=\pi_{1}$. Let $C \in \pi_{2}$ be such that $C \subseteq B$. Let $G$ be a maximal subgroup of $G_{B}$ that contains $G_{C}$. We define

$$
\pi_{13}=\left(\pi_{1} \backslash\{p B: p \in P\}\right) \cup\{p G C: p \in P\} .
$$

It is easy to see that $\pi_{13}$ is a remainder of the form $\left(n_{1}, J_{1}, m_{1}, r_{1}\right)$ and $\pi_{1} \supseteq \pi_{13} \supseteq$ $\pi_{2}$. Since $\pi_{13} \neq \pi_{1}$, we have $\pi_{13}=\pi_{2}$, and we have case (5)(a).

Now we assume that $\pi_{12}=\pi_{2}$. Let $C \in \pi_{2}$ such that $C \subseteq B$. We have $C^{B}=C$. Hence, $G_{C}=G_{B}$ and $B=C_{1} \cup C_{n}$, where $n>1$. Let $A^{i}$ be a maximal antichain in $C_{i}$. It is easy to see that $\bigcup_{i=1}^{n} A_{i}$ is a maximal antichain in $B$. Define $A_{1}=A^{1}$ and $A_{2}=\bigcup_{i=2}^{n} A^{i}$. We define $\pi_{14}$ as follows:

$$
\pi_{14}=\left(\pi_{1} \backslash\{p B: p \in P\}\right) \cup\left\{p G_{B} A_{1}: p \in P\right\} \cup\left\{p G_{B} A_{2}: p \in P\right\} .
$$

It is not difficult to see that $\pi_{14}$ is a remainder of the form $\left(n_{1}, J_{1}, m_{1}, r_{1}\right)$. Moreover, $\pi_{1} \supseteq \pi_{14} \supseteq \pi_{2}$ and $\pi_{14} \neq \pi_{1}$. Therefore, $\pi_{14}=\pi_{2}$, and we have cases $(5)(\mathrm{b})$. This completes the proof of the theorem.

\section{FIRST-ORDER DEFINABILITY}

The second part of the paper is devoted to showing one possible application of the description of the lattice $L(S P)$ developed in the previous sections. We prove that every equational theory of strongly permutative semigroups is individually definable (Theorem 10.9). We start with the following.

Theorem 7.1. The set $\mathcal{L}(C o m)$ is definable in $\mathcal{L}(S P)$.

Proof. To this end, we have a look at the upper part of the lattice $L(S P)$. The greatest element in $\mathcal{L}(S P)$ is theory $T(2,[(1)], 0,1, \emptyset)$ of all equations. There are the following co-atoms in $\mathcal{L}(S P)$ :

- $S=T(2,[(x)], 1,1, \emptyset)$, the theory of semilattices; 
- $A_{p}=T(2,[(x)], 0, p, \emptyset)$, the theory of Abelian groups of prime exponents, for all primes $p \geq 2$;

- $N=T(2,[(x y)], 0,1, \Theta)$ of semigroups with zero multiplication, defined by $x y=u z$.

Obviously the set of co-atoms is definable.

Let $M$ denote an equational theory of strongly permutative semigroups generated by the equation $x y=y x$. This is the smallest theory that belongs to $L(C o m)$. It is well known (see [3], for instance) that the theory $M$ is a meeting of all theories $A_{p}$. Since $M \subset S \cap N, M$ is a meeting of all atoms of $\mathcal{L}(S P)$. Thus, $M$ is definable.

$L(C o m)$ is the set of all theories of $\mathcal{L}(S P)$ that are greater than or equal to $M$.

An immediate consequence is

Corollary 7.2. Every set of theories and every individual theory that is definable in $\mathcal{L}(C o m)$ is definable in $\mathcal{L}(S P)$, too.

A theory $T$ is what we call a balanced theory if it consists of the equations of the form $y_{1} \cdots y_{k}=y_{\sigma(1)} \cdots y_{\sigma(k)}$, where $y_{i}$ are not necessarily different. In other words an equation is balanced if every letter occurs in both its sides the same number of times.

Corollary 7.3. The set $\mathcal{L}(B a l)$ of strongly permutative balanced theories is definable in $\mathcal{L}(S P)$.

Proof. $M$ is the greatest balanced theory. Since $M$ is definable, the set of balanced theories $\mathcal{L}(\mathrm{Bal})$ is definable as a set of theories that are less than or equal to $M$.

In addition, we have

Corollary 7.4. The parameters $m$ and $r$ are definable.

Proof. These parameters are definable in $L(\mathrm{Com})$ (see [13]). Now, theories that have given $m$ and $r$ are those theories $T$ where the theory $T \vee M$ has parameters $m$ and $r$.

\section{Definability of the Parameter $n$}

In this section, we show that, for a given $n \geq 2$, the set of the theories $T \in \mathcal{L}(S P)$, such that $n(T)=n$ is definable.

Note that if $T$ is a balanced theory, then we have $J(T)=\emptyset, m(T)=\infty$, and $r(T)=\infty$. This means, in particular, that every dual-cover of a given balanced theory has the form as in Theorem 6.4(1) or (5). We recall that for $G<S_{n}$ and variables $y_{1}, \ldots, y_{n}$ (not necessarily different), by $G\left\{y_{1} \cdots y_{n}\right\}$ we denote the block $\left\{y_{g(1)} \cdots y_{g(n)}: g \in G\right\}$. We say that the block $K$ generates a theory $T$ if $T$ is generated by all the equations $(v, w)$, where $v, w \in K$. In the sequel, we assume that the variables $x_{i}$ and $x_{j}$, where $i \neq j$, are different unless we say otherwise. By $\operatorname{Bal}(n)$, we denote the theory generated by the block $S_{n}\left\{x_{1} \cdots x_{n}\right\}$ (when, recall, $\left\{x_{1} \cdots x_{n}\right\}$ is a one-element antichain).

The greatest balanced theory is $M=\operatorname{Bal}(2)$. It consists of all the balanced equations and is generated by the equation $x y=y x$. It has the only one co-atom $\operatorname{Bal}(3)$, which is obviously definable. 
We wish to show that all the theories $\operatorname{Bal}(n)$ are definable. We do it inductively. We know that theories $\operatorname{Bal}(2)$ and $\operatorname{Bal}(3)$ are definable. Assume that $\operatorname{Bal}(n)$, $n \geq 3$, is definable. We show that $\operatorname{Bal}(n+1)$ is definable. Dual covers of $\operatorname{Bal}(n)$ are theories $B_{G}$ generated by the blocks:

- $G\left\{x_{1} \cdots x_{n}\right\}$,

- $S_{n}\{w\}$, where $w$ is $\leq_{n}$-equivalent with $x_{1}^{2} x_{2} \cdots x_{n-1}$,

- $S_{n+1}\left\{x_{1} \cdots x_{n+1}\right\}$,

where $G$ is a maximal subgroup of $S_{n}$. Obviously, the set of dual covers of $\operatorname{Bal}(n)$ is definable.

It is well known (see [2, for instance) that $S_{n}$ has the following maximal subgroups:

- $S_{I} \times S_{J}$, where $I, J$ is a partition of the set $\{1, \ldots, n\}$ such that $|I| \neq|J|$,

- $S_{k} W r S_{t}$, for a partition $I_{1}, \ldots, I_{k}$ of the set $\{1, \ldots, n\}$ such that $\left|I_{i}\right|=t$ for every $1 \leq i \leq k$ and $k t=n$,

- a maximal primitive subgroup of $S_{n}$.

As usual, by $A_{n}$ we denote the alternating group consisting of even permutations of the set $\{1, \ldots, n\}$. We say that a group $G \subseteq S_{n}$ has an inner fixed point interval if there is $1<i<n$ such that $G$ preserves the sets $\{1, \ldots, i-1\}$ and $\{i+1, \ldots, n\}$, and moreover, the action of $G$ is nontrivial on each of these sets.

The following result was proved in [19] and also in [15, with another proof. We prefer the formulation from [15. Unfortunately, it contains a small gap. The correct formulation is the following.

Theorem 8.1 ([19, 15]). Let $T$ be a balanced theory such that $G\left\{x_{1} \cdots x_{n}\right\}$ is a block containing $x_{1} \cdots x_{n}$ and $H\left\{x_{1} \cdots x_{n+1}\right\}$ is a block containing $x_{1} \cdots x_{n+1}$. Then $H=S_{n+1}$ or $H=A_{n+1}$ if and only if $G$ has no inner fixed point intervals and is not a subgroup of $S_{5}$ generated by a permutation $(1,4)(2,5)$. In such a case $H=A_{n+1}$ if and only if the set of odd numbers is fixed by $G$ and $G \subseteq A_{n}$.

Observe that the subgroup of $S_{5}$ generated by the permutation $(1,4)(2,5)$ is not maximal. Moreover, the permutation group $A_{n}$ contains a permutation $\sigma$ such that $\sigma(k)-k$ is odd for some $k$. In addition, every other maximal subgroup of $S_{n}$ contains an odd permutation. Therefore, by Theorem 8.1, we obtain that, except for the permutation groups $S_{\{1\}} \times S_{\{2, \ldots, n\}}$ and $S_{\{1, \ldots, n-1\}} \times S_{\{n\}}$, the equations generated by the block $S_{n+1}\left\{x_{1} \cdots x_{n+1}\right\}$ are consequences of the equations generated by the block $G\left\{x_{1} \cdots x_{n}\right\}$ for a maximal subgroup $G$ of $S_{n}$. In the two exceptional cases the consequences are equations generated by the blocks $S_{\{1\}} \times S_{\{2, \ldots, n+1\}}\left\{x_{1} \cdots x_{n+1}\right\}$ and $S_{\{1, \ldots, n\}} \times S_{\{n+1\}}\left\{x_{1} \cdots x_{n+1}\right\}$, respectively.

By the remark above, if $G$ is neither $S_{\{1\}} \times S_{\{2, \ldots, n\}}$ nor $S_{\{1, \ldots, n-1\}} \times S_{\{n\}}$, then a theory $B_{G}$ has at most one dual cover that is not contained in another theory of the form $B_{H}$. If there is such a dual cover, then it is generated by the blocks listed below:

- $G\left\{x_{1} \cdots x_{n}\right\}$,

- $S_{n+1}\left\{x_{1} \cdots x_{n+1}\right\}$, and for $n>3$

- $S_{n}\{w\}$, where $w$ is $\leq_{n}$-equivalent either to the word $x_{1}^{3} x_{2} \cdots x_{n-2}$ or to the word $x_{1}^{2} x_{2}^{2} x_{3} \cdots x_{n-2}$.

We denote this dual cover $C_{G}$. 
For $G \in\left\{S_{\{1\}} \times S_{\{2, \ldots, n\}}, S_{\{1, \ldots, n-1\}} \times S_{\{n\}}\right\}$ there are two dual covers of $B_{G}$ that are not contained in another theory of the form $B_{H}$. One is as in the case above, and the second is generated by the blocks:

- $G\left\{x_{1} \cdots x_{n}\right\}$,

- $S_{n}\{w\}$, where $w$ is $\leq_{n}$-equivalent to the word $x_{1}^{2} x_{2} \cdots x_{n-1}$,

- $S_{n+2}\left\{x_{1} \cdots x_{n+2}\right\}$.

We denote it $D_{G}$.

This implies that the sets

$$
\left\{B_{G}: G \in\left\{S_{\{1\}} \times S_{\{2, \ldots, n\}}, S_{\{1, \ldots, n-1\}} \times S_{\{n\}}\right\}\right\}
$$

and

$$
\left\{B_{G}: G \notin\left\{S_{\{1\}} \times S_{\{2, \ldots, n\}}, S_{\{1, \ldots, n-1\}} \times S_{\{n\}}\right\}\right\}
$$

are definable.

We show that, in both cases where $G \in\left\{S_{\{1\}} \times S_{\{2, \ldots, n\}}, S_{\{1, \ldots, n-1\}} \times S_{\{n\}}\right\}$, we can distinguish between $C_{G}$ and $D_{G}$. Indeed, in both of these cases $D_{G}$ has two dual covers that are not under $B_{H}$ for $H \neq G$. We list them for the group $S_{\{1\}} \times S_{\{2, \ldots, n\}}$ (the second case is analogous):

(1) the theory generated by the blocks:

- $G\left\{x_{1} \cdots x_{n}\right\}$,

- $S_{n}\{w\}$, where $w$ is $\leq_{n}$-equivalent to the word $x_{1}^{2} x_{2} \cdots x_{n-1}$,

- $S_{n+3}\left\{x_{1} \cdots x_{n+3}\right\}$;

(2) the theory $F_{S_{\{1\}} \times S_{\{2, \ldots, n\}}}$ generated by the blocks:

- $G\left\{x_{1} \cdots x_{n}\right\}$,

- $S_{n+2}\left\{x_{1} \cdots x_{n+2}\right\}$,

- $S_{\{1\}} \times S_{\{2, \ldots, n+1\}}\{w\}$, where $w$ is $\leq_{n}$-equivalent to the word $x_{1} x_{2}^{2} \cdots x_{n}$, and for $n>3$,

- $S_{n}\{w\}$, where $w$ is $\leq_{n}$-equivalent either to the word $x_{1}^{3} x_{2} \cdots x_{n-2}$ or with the word $x_{1}^{2} x_{2}^{2} x_{3} \cdots x_{n-2}$.

For theories $C_{G}$, we have two cases. For $n=3, C_{G}$ has the only one dual cover that is not under $B_{H}$ for $H \neq G$. This is $F_{G}$. For $n>3, C_{G}$ has three of them. Again, we list them for the group $S_{\{1\}} \times S_{\{2, \ldots, n\}}$ :

(1) the theory $F_{G}$,

(2) the theory generated by the blocks:

- $G\left\{x_{1} \cdots x_{n}\right\}$,

- $S_{n+1}\left\{x_{1} \cdots x_{n+1}\right\}$, and for $n>3$

- $S_{n}\{w\}$, where $w$ is $\leq_{n}$-equivalent either to the word $x_{1}^{3} x_{2} \cdots x_{n-2}$ or (for $n \geq 6$ ) to the word $x_{1}^{2} x_{2}^{2} x_{3}^{2} x_{4} \cdots x_{n-3}$,

(3) the theory generated by the blocks:

- $G\left\{x_{1} \cdots x_{n}\right\}$,

- $S_{n+1}\left\{x_{1} \cdots x_{n+1}\right\}$, and for $n>3$

- $S_{n}\{w\}$, where $w$ is $\leq_{n}$-equivalent to the word $x_{1}^{2} x_{2}^{2} x_{3} \cdots x_{n-2}$.

Consequently, the set $\left\{C_{G}: G \in\left\{S_{\{1\}} \times S_{\{2, \ldots, n\}}, S_{\{1, \ldots, n-1\}} \times S_{\{n\}}\right\}\right\}$ is definable.

For $\left.G \notin\left\{S_{\{1\}} \times S_{\{2, \ldots, n\}}, S_{\{1, \ldots, n-1\}} \times S_{\{n\}}\right\}\right\}$, by $E_{G}$ we denote the minimal theory which is under $B_{G}$ and is not under $B_{H}$ for $H \neq G$. For $G \in\left\{S_{\{1\}} \times\right.$ 
$\left.S_{\{2, \ldots, n\}}, S_{\{1, \ldots, n-1\}} \times S_{\{n\}}\right\}$, by $E_{G}$ we denote the minimal theory which is under $C_{G}$ and is not under $B_{H}$ for $H \neq G$. It is clear that $E_{G}$ is generated by the blocks:

- $G\left\{x_{1} \cdots x_{n}\right\}$,

- $S_{n+1}\left\{x_{1} \cdots x_{n+1}\right\}$.

Obviously, the set consisting of all the theories $E_{G}$ is definable. Therefore, the meet of $E_{G}$ is definable. We show that this meet is equal to the theory $\operatorname{Bal}(n)$. In fact, this is enough to take the meet of those $G$ that have a form $S_{\{i\}} \times S_{\{1, \ldots, n\} \backslash\{i\}}$.

Fix a word $w=y_{1} \cdots y_{n}$. Then a block containing $w$ in $E_{G}$, for $G=S_{\{1, \ldots, n\} \backslash\{i\}}$, has the form $G(w)$. This mean that if a word $v=z_{1} \cdots z_{n}$ belongs to the block $G(w)$, then $v$ is $\leq_{n}$-equivalent with $w$ and $v=\sigma(w)$ for some $\sigma \in G$. In particular, $z_{i}=y_{i}$. Therefore, the block of $w$ in a theory $\bigwedge E_{G}$ contains only one element, $w$. This means that $\bigwedge E_{G} \subseteq \operatorname{Bal}(n+1)$. Obviously, the opposite inclusion also holds. Consequently, $\operatorname{Bal}(n+1)$ is definable. Thus, by induction, $\operatorname{Bal}(n)$ is definable for every $n \geq 2$.

Corollary 8.2. For given $n \geq 2$, the set of theories of $\mathcal{L}(S P)$ of the form $T(n, J, m, r, \pi)$ is definable (the parameter $n$ is definable).

Proof. This is the set of those theories which contain the theory $\operatorname{Bal}(n)$ and does not contain the theory $\operatorname{Bal}(n-1)$.

For a given $n, m$, and $r$, we denote $S(n, m, r)$ to be the set of all theories of the form $T(n, J, m, r, \pi)$.

Corollary 8.3. Every set $S(n, m, r)$ is definable.

\section{Definability of the Parameter $J$}

Let $\Theta$ denote the trivial remainder (i.e., consisting of the trivial equations only). As a next step, in this section we show that for a given $n, J, m$, and $r$, the theory $T(n, J, m, r, \Theta)$ is definable, up to duality. Such theories are called Schwabauer theories. In view of earlier results, it is the same as showing that every order filter $J$ is definable, up to duality. We start with those filters of the bqo $\leq_{n}$ that are generated by a single word.

Fix $n \geq 2$. Let $Q(n, 0,1)$ be a set of those theories $T \in S(n, 0,1)$ for which there is exactly one dual cover of $T$ that belongs to the set $S(n, 0,1)$. Obviously, the set $Q(n, 0,1)$ is definable. It is clear that if $T \in Q(n, 0,1)$, then $T$ is generated by the block $S_{n}\left\{x_{1} \cdots x_{n}\right\}$, where $x_{i} \neq x_{j}$ for $i \neq j$, and one additional equation $e=(w, v)$, which is not balanced (otherwise $m=r=\infty$ and $T \notin Q(n, 0,1)$ ). We consider a few cases.

Since $m\left(e^{\prime}\right)>0$ for every regular equation $e^{\prime}$, we may consider only irregular equations. Then assume that $e$ is irregular and moreover that $v$ and $w$ are incomparable. If it happens that $r(e)=1$, then, by Lemma 5.1 we have that $T=T(n, J, 0,1, \Theta)$, where $J=[v, w]$. However, this theory has two dual covers that belong to $S(n, 0,1)$. They have the forms $T\left(n, J_{v}, 0,1, \pi_{1}\right)$ and $T\left(n, J_{w}, 0,1, \pi_{2}\right)$ for some $\pi_{1}$ and $\pi_{2}$, where $J_{u}=J \backslash\left\{u^{\prime}: u^{\prime}\right.$ is $\leq_{n}$-equivalent with $\left.u\right\}$. Therefore, $T \notin Q(n, 0,1)$.

Now let $v$ and $w$ be comparable. We may assume $v \leq_{n} w$. Let $J=[v]$. If $r(e)=1$, then by Lemma $5.1, T=T(n, J, 0,1, \Theta)$. Moreover, $T$ has exactly one dual cover that belongs to $S(n, 0,1)$. It has the form $T\left(n, J_{v}, 0,1, \pi\right)$ for some $\pi$. It remains to show that, for every word $v$, there exists a word $w \geq_{n} v$ such that 
$e=(v, w)$ is irregular and $r(e)=1$. Take $w=v y$, where $y$ is a variable that does not occur in $\operatorname{supp}(v)$. Then, $w$ is as required. This implies that the set $S(n, 0,1)$ is a set of those theories of the form $T(n, J, 0,1, \Theta)$ for which $J$ is generated by a single word.

For a fixed $n \geq 2, m \geq 0$, and $r>0$ such that either $m>0$ or $r>1$, we define $Q(n, m, r)$ to be the set of all theories of the form $T(n, J, m, r, \Theta)$, where $J$ is generated by a single word.

Corollary 9.1. All the sets $Q(n, m, r)$ are definable.

Proof. Assume that either $m>0$ or $r>1$. Let $T\left(n, J_{m}, m, r, \Theta\right)$ be a theory, where $J_{m}$ is a filter in a bqo $\leq_{n}$ consisting of all the words $w$ such that $m(w) \geq m$. Then, $T\left(n, J_{m}, m, r, \Theta\right)$ is the largest element in $S(n, m, r)$, and therefore, $T\left(n, J_{m}, m, r, \Theta\right)$ is definable. The set $Q(n, m, r)$ consists of all those theories $T$ that belong to the set $S(n, m, r)$ and for which there exists a theory $T^{\prime} \in Q(n, 0,1)$ such that $T=T^{\prime} \wedge T\left(n, J_{m}, m, r, \Theta\right)$. Therefore, the set $Q(n, m, r)$ is definable.

Now we have a natural order preserving "one to one" correspondence between the set of the classes of equivalent words in a bqo $\left(W, \leq_{n}\right)$ and the set $Q(n, 0,1)$. Proving definability of an element (set) in one of these bqo's is the same as proving definability of the corresponding element (set) in the other. To simplify the notation, we will write $w$, also, for the equivalence class containing the word $w$. Moreover, it is clear that if a class $w$ is definable in $\left(W, \leq_{n}\right)$, for some $n$, then $w$ is definable in $\left(W, \leq_{n^{\prime}}\right)$, for every $n^{\prime} \leq n .\left(T\left(n^{\prime},[w], 0,1, \Theta\right)\right.$ is a join of $T(n,[w], 0,1, \Theta)$ and $\operatorname{Bal}\left(n^{\prime}\right)$.) The same is true for definable sets of classes. Therefore, we may always assume that $n$ is suitably large. We will do it without further mention. We also assume, without further mention, that the letters with different names are different, unless we say otherwise.

Now we show that in every bqo $\left(W, \leq_{n}\right)$, each class is definable up to duality (the reversing of letters). For a class $w$, by $[w]$, we denote the set of those classes $v$ such that $\operatorname{supp}(v)=\operatorname{supp}(w)$ and $|v|_{x}=|w|_{x}$ for every $x \in \operatorname{supp}(w)$. First, we use the fact (proved in [14, Theorem 4.3]) that each element in the bqo $\left(W, \leq_{2}\right)$ is definable. This implies that, for every $n$, each set $[w]$ is definable in $\left(W, \leq_{n}\right)$ (it consists of a single class $w$ for $n=2)$. Moreover, if $w \in\left\{x^{k}, x_{1} \cdots x_{k}\right.$ : where $x_{i}$ are all different and $\left.k \in \mathbb{N}_{+}\right\}$, then $[w]=\{w\}$. Thus, in these cases $w$ is definable.

Consider the set $\left[x^{2} y\right]$. It consists of three classes: $x^{2} y, y x^{2}$, and $x y x$. The first two are $\leq_{n}$ greater than $x^{2}$. The other is not. Therefore $x y x$ is definable. The elements $x^{2} y$ and $y x^{2}$ are the smallest undefinable elements. However, they are definable, up to duality. We show that, if we could distinguish between those two elements, we would define every class in $\left(W, \leq_{n}\right)$. This will show that each element is definable up to duality. We assume now that in $\left(W, \leq_{n}\right)$ we are able to single out element $x^{2} y$. We show that we are able to define every element.

For a fixed $k \geq 2$, the element $x^{2} y_{1} \cdots y_{k}$ is the only element belonging to the set $\left[x^{2} y_{1} \cdots y_{k}\right]$ that is a cover of the class $x^{2} y_{1} \cdots y_{k-1}$ and is not greater than the class $y x^{2}$. Then, by induction on $k$, the class $x^{2} y_{1} \cdots y_{k}$ is definable for every $k$. Similarly, the elements $y_{1} \cdots y_{k} x^{2}, k \geq 1$, are definable.

Fix $k \geq 3$. The element $x^{k} y$ is the only member of the set $\left[x^{k} y\right]$ that is greater than the classes $x^{k}, x^{2} y_{1} \cdots y_{k-1}$ but not greater than the class $y_{1} \cdots y_{k-1} x^{2}$. Hence, $x^{k} y$ is definable and, similarly, $y x^{k}$ is definable. For $l \geq 2$, an element $x^{k} y_{1} \cdots y_{l}$ is the only member of $\left[x^{k} y_{1} \cdots y_{l}\right]$ that is greater than $x^{k} y_{1} \cdots y_{l-1}$ but not greater 
than $y x^{k}$. By induction, all elements $x^{k} y_{1} \cdots y_{l}$ are definable. Similarly, the same is true for elements $y_{1} \cdots y_{l} x^{k}$. For fixed $k \geq 2, l \geq 1$, and $j \geq 1$, the element $y_{1} \cdots y_{l} x^{k} z_{1} \cdots z_{j}$ is the only member of the set $\left[y_{1} \cdots y_{l} x^{k} z_{1} \cdots z_{j}\right]$ that is greater than both $x^{k} z_{1} \cdots z_{j}$ and $y_{1} \cdots y_{l} x^{k}$.

For $j, k, l \geq 0$, we denote $w(j, k, l)$ to be the word $y_{1} \cdots y_{j} x z_{1} \cdots z_{k} x t_{1} \cdots t_{l}$. Then the class $w(j, k, l)$ is the only member of the set $[w(j, k, l)]$ that is smaller than $y_{1} \cdots y_{j} x^{k+2} t_{1} \cdots t_{l}$ and is not smaller than $y_{1} \cdots y_{j+1} x^{k+1} t_{1} \cdots t_{l}$ or than $y_{1} \cdots y_{j} x^{k+1} t_{1} \cdots t_{l+1}$. Thus, the classes $w(j, k, l)$ are definable.

Finally, let $w=x_{1} \cdots x_{k}$ be an arbitrary word where not all $x_{i}$ are different. Then the class $w$ is the only member of the set $[w]$ that is greater than the classes $w(i-1, j-i-1, k-j)$ for all $1 \leq i<j \leq k$ such that $x_{i}=x_{j}$. Thus, we have the following.

Lemma 9.2. For every $n \geq 2$, each class is definable in the bqo $\left(W, \leq_{n}\right)$ with the element $x^{2} y$ singled out.

This immediately implies the following.

Theorem 9.3. For every $n \geq 2$, each class is definable in the bqo $\left(W, \leq_{n}\right)$, up to duality. Moreover, a class $w$ is definable if and only if the word $w$ is symmetric.

Since for every $n \geq 2$ each filter $J$ of the bqo $\left(Q, \leq_{n}\right)$ is finitely generated, we have the following.

Corollary 9.4. For a given $n, J, m$, and $r$, the theory $T(n, J, m, r, \Theta)$ is definable, up to duality. Moreover, the set of theories of the form $T(n, J, m, r, \pi)$ is definable, up to duality (the parameter $J$ is definable, up to duality).

\section{Definability of REgUlar EQUATIONS}

In the previous section, we have proved that every Schwabauer theory is definable. Since every irregular equation generates a Schwabauer theory, we can restrict our investigation to the theories generated by regular equations. Moreover, if a regular equation $e=(v, w)$ is such that $v<_{n} w$, then $e$ also generates a Schwabauer theory. Therefore, we may restrict ourselves to the theories generated by a regular equation $e=(v, w)$, where either $w=p(v)$ for some permutation $p$ (a substitutive equation) or $v$ and $w$ are $\leq_{n}$ incomparable (a parallel equation).

Generally, this part is harder than the previous one. Yet, in this case we can follow exactly some arguments applied in [14 and [6]. Therefore, we only point out corresponding proofs and give some additional comments, if necessary. First, we observe that we can recognize $e$ up to permutation.

Theorem 10.1. Given $\leq_{n}$ incomparable words $v$ and $w$, the following properties are definable in $\mathcal{L}(S P)$ (up to duality):

(i) an equation $(v, p(v)) \in T$ for some permutation $p$,

(ii) $T$ is generated by a set of equations of the form $(v, p(v))$ and the block $S_{n}\left\{x_{1} \cdots x_{n}\right\}$

(iii) an equation $(v, p(w)) \in T$ for some permutation $p$,

(iv) $T$ is generated by a set of equations of the form $(v, p(w))$ and the block $S_{n}\left\{x_{1} \cdots x_{n}\right\}$.

Proof. The proof is exactly the same as the proofs of [14, Theorem 5.1 and Theorem 5.4]. 
For every $k$, any subgroup of $S_{k}$ which is not cyclic of prime power order, has at least two (inclusion) incomparable subgroups. Hence, we may improve Theorem 10.1 by taking the theories of the form (ii) that do not have two incomparable smallest theories generated by the sets of equations of the form as in (ii). In case (iv), we simply take the smallest theories of this form.

Corollary 10.2. Given $\leq_{n}$ incomparable words $v$ and $w$, the following properties are definable in $\mathcal{L}(S P)$ (up to duality):

(i) $T$ is generated by an equation of the form $(v, p(v))$ and the block $S_{n}\left\{x_{1} \cdots x_{n}\right\}$, where the order of the permutation $p$ is a prime power,

(ii) $T$ is generated by an equation of the form $(v, p(w))$ and the block $S_{n}\left\{x_{1} \cdots x_{n}\right\}$.

10.1. Theories generated by substitutive equations. We proceed to prove that every theory in $\mathcal{L}(S P)$ is definable (up to duality). We consider these theories that are generated by an equation $(v, p(v))$ and the block $S_{n}\left\{x_{1} \cdots x_{n}\right\}$. We may assume that $n$ is large enough (i.e., $n>|v|+1$, for instance). For a fixed $n$, a word $v$ and a permutation $p$, we define $T(n,(v, p(v)))$ to be the theory generated by an equation $(v, p(v))$ and the block $S_{n}\left\{x_{1} \cdots x_{n}\right\}$.

Lemma 10.3. Let $v=v\left(x_{1}, \ldots, x_{k}\right), p \in S_{k}$, and $T=T(n,(v, p(v)))$. Let $i \neq j$, $s \neq t$ be fixed. Then there exists $\sigma=p^{l}$ for some l such that $\{\sigma(i), \sigma(j)\}=\{s, t\}$ if and only if there is $q \in S_{k-1}$ such that $\left(g_{x_{i} x_{j}}(v), q\left(g_{x_{s} x_{t}}(v)\right)\right) \in T$.

Proof. Exactly the same as the proof of [6, Lemma 5.1].

Now we may prove the following.

Theorem 10.4. Every theory of the form $T(n,(v, p(v)))$, where the order of $p$ is a prime power, is definable (up to duality).

Proof. Using Lemma 10.3, exactly the same as the proof of [6, Theorem 5.3]. Note however that, since $n$ is large enough, the word $v$ has no inner symmetries. Therefore, the case is similar to the case in [6, where terms of the chain are different from each other. This offers the possibility to simplify the proof considerably.

As a consequence, we immediately have the following.

Theorem 10.5. A theory of the form $T(n,(v, p(v)))$ is definable (up to duality) for every $n \geq 2$, a word $v$, and a permutation $p$ of $\operatorname{supp}(v)$.

Proof. It is enough to observe that $T(n,(v, p(v)))=\operatorname{Bal}(n) \vee \bigvee T\left(m,\left(v, p_{i}(v)\right)\right)$, where $m$ is large enough, $p=p_{1} \cdots p_{k}$, and an order of each $p_{i}$ is a prime power.

10.2. Theories generated by parallel equations. The last case to consider is that of the theories generated by an equation $(v, w)$ and the block $S_{n}\left\{x_{1} \cdots x_{n}\right\}$, where the equation $(v, w)$ is regular and $v$ and $w$ are incomparable. We may assume again that $n$ is large enough (i.e., $n>\max (|v|,|w|)+1$, for instance). For a fixed $n$ and words $v$ and $w$ such that the equation $(v, w)$ is parallel, we define $T(n,(v, w))$ to be a theory generated by an equation $(v, w)$ and the block $S_{n}\left\{x_{1} \cdots x_{n}\right\}$.

We are going to prove that every theory of the form $T(n,(v, w)$ ) is definable (up to duality). We start with the case when $|\operatorname{supp}(v)| \geq 3$.

Lemma 10.6. Let $(v, w)$ be a parallel equation such that $|\operatorname{supp}(v)| \geq 3$. Then $T(n,(v, w))$ is definable (up to duality). 
Proof. The proof is exactly the same as [12, Lemma 4.7]. Note that the proof of [12, Lemma 4.7] uses only [12, Lemma 4.6], which we do not need, since we have Theorem 10.5. In particular, the proof does not use [12, Lemma 4.4].

Alternatively, it is also possible to use the proof of [6, Lemma 6.2] in the easier version, since for $n$ large enough, the words $v$ and $w$ have no inner symmetries.

It remains to prove the similar lemma with assumption $|\operatorname{supp}(v)|=2$. This is different from the commutative case of [6]. We are not able to follow [12, Lemma 4.4], since it uses the fact that the theory generated by the equation $(x, x y)$ is definable (up to duality). In the present case, we have an additional commutativity which begins from some level $n$, and therefore, the equation $(x, x y)$ generates the theory of all equations. Yet the following lemma settles the problem.

Lemma 10.7. Let $(v, w)$ be a parallel equation such that $|\operatorname{supp}(v)|=2$. Then $T(n,(v, w))$ is definable (up to duality).

Proof. Let $v=v(x, y), w=w(x, y)$, and $z \notin\{x, y\}$. We consider an equation $e=(v z, w z)$. It is clear that the equation $e$ is parallel and $\operatorname{supp}(v z)=3$. Hence, by Lemma 10.6, the theory $T(n, e)$ is definable. Obviously, $e$ is a consequence of the equation $(v(x, y), w(x, y))$ and therefore, $T(n,(v(x, y), w(x, y))) \supset T(n, e)$. Since $n$ is large enough, $v z, w z \notin J(T(n,(v(x, y), w(y, x))))$. This implies that $T(n, e) \nsubseteq T(n,(v(x, y), w(y, x)))$. Hence, we can distinguish between theories $T(n,(v(x, y), w(x, y)))$ and $T(n,(v(x, y), w(y, x)))$. Thus, both those theories are definable (up to duality).

Using Lemma 10.6 and Lemma 10.7, we can prove the following.

Corollary 10.8. A theory $T(n,(v, w)$ ) is definable (up to duality) for every $n \geq 2$ and each parallel equation $(v, w)$.

Proof. It is enough to observe that $T(n,(v, w))=\operatorname{Bal}(n) \vee T(m,(v, w))$, where $m$ is large enough.

Combining the results from sections $7-10$ and using Theorem [5.10, we have the result announced at the beginning of section 7 .

Theorem 10.9. Every equational theory of strongly permutative semigroups is individually first-order definable, up to duality, in the lattice of equational theories of strongly permutative semigroups.

\section{REFERENCES}

[1] J. Almeida, Some order properties of the lattice of varieties of commutative semigroups, Canad. J. Math. 38 (1986), no. 1, 19-47. MR835034(87h:20108)

[2] J. D. Dixon and B. Mortimer, Permutation Groups, Springer-Verlag (GTM 163) 1996. MR 1409812 (98m:20003)

[3] T. Evans, The lattice of semigroup varieties, Semigroup Forum 2 (1971), 1- 43. MR0284528 $(44: 1753)$

[4] M. Grech, Irreducible varieties of commutative semigroups, J. Algebra 261 (2003), 207-228. MR.1967162 (2004a:20067)

[5] M. Grech, Well- and better-quasi-orderings in the lattice of varieties of commutative semigroups, International Journal of Algebra and Computation 17 (2007), 869-879. MR2340821 (2008f:20146)

[6] M. Grech, Automorphisms of the lattice of equational theories of commutative semigroups, Trans. Amer. Math. Soc. 361 (2009), 3435-3462. MR2491887|(2009k:08006) 
[7] M. Grech, A. Kisielewicz, Covering relation for equational theories of commutative semigroups, J. Algebra 232 (2000), 493-506. MR.1792743 (2002g:20102)

[8] J. Ježek, The lattice of equational theories I: Modular elements, Czech. Math. J. 31 (1981), 127-152. MR604120 (84e:08007a)

[9] J. Ježek, The lattice of equational theories II: The lattice of full sets of terms, Czech. Math. J. 31 (1981), 573-603. MR631604 (84e:08007b)

[10] J. Ježek, The lattice of equational theories III: Definability and automorphisms, Czech. Math. J. 32 (1982), 129-164. MR646718 (84e:08007c)

[11] J. Ježek, The lattice of equational theories IV: Equational theories of finite algebras, Czech. Math. J. 36 (1986), 331-341. MR831318 (87g:08015)

[12] J. Ježek, R. McKenzie, Definability in the lattice of equational theories of semigroups, Semigroup Forum 46 (1993), 199-245. MR.1200214 (94a:03052)

[13] A. Kisielewicz, Varieties of commutative semigroups, Trans. Amer. Math. Soc. 342 (1994), 275-306. MR1211411 (94j:20065)

[14] A. Kisielewicz, Definability in the lattice of equational theories of commutative semigroups, Trans. Amer. Math. Soc. 356 (2004), 3483-3504. MR2055743 (2005a:08011)

[15] A. Kisielewicz, Permutability class of a semigroup, J. Algebra 226 (2000), 295-310. MR:1749890 (2001c:20122)

[16] R. McKenzie, Definability in the lattice of equational theories, Annals of Math. Logic 3 (1971), 197-237. MR0280349 (43:6069)

[17] C. St. J. A. Nash-Williams, On well-quasi-ordering infinite trees, Proc. Camb. Phil. Soc. 61 (1965), 697-720. MR0175814(31:90)

[18] P. Perkins, Bases for equational theories of semigroups, J. Algebra 11 (1969), 298-314. MR0233911 (38:2232)

[19] G. Pollák, On the consequences of permutation identities, Acta Sci. Math. (Szeged) 34 (1973), 323-333. MR0322084 (48:448)

[20] M. S. Putcha and A. Yaqub, Semigroups satisfying permutation identities, Semigroup Forum $3(1971 / 72)$, no. 1, 68-73. MR0292969 (45:2050)

[21] O. Sapir, Finitely generated permutative varieties, Semigroup Forum 78 (2009), 427-449. MR2511777 (2010d:20061)

[22] A. Tarski, Equational logic and equational theories of algebras, Contributions to Mathematical Logic (Hanover 1966), 275-288, North-Holland, Amsterdam, 1968. MR0237410 (38:5692)

Institute of Mathematics, University of WrocŁaw, Pl. Grunwaldzki 2, 50-384 Wroceaw, Poland

E-mail address: Mariusz.Grech@math.uni.wroc.pl 